\title{
Brachial-ankle pulse wave velocity, cardio-ankle vascular index, and prognosis
}

This article was published in the following Dove Press journal:

Vascular Health and Risk Management

\section{Dai Ato \\ Gakujutsu Shien Co., Ltd, Tokyo, Japan}

Correspondence: Dai Ato

Gakujutsu Shien (Academic Support) Co., Ltd., 2-I-I7 Nihonbashi, Chuo-ku, Tokyo 103-0027, Japan

Tel +8I 368696438

Email ato.dail@wind.ocn.ne.jp
Background: Brachial-ankle pulse wave velocity (baPWV) and cardio-ankle vascular index (CAVI) are indices of arterial stiffness, and several studies have used these indices. However, there is no comprehensive review of these parameters in the prognostic significance.

Methods: The aim of this study was to review the articles exploring the prognostic significance of these parameters. Articles demonstrating independent significance after multivariate analysis on the Cox proportional hazards model were defined as "successful." The success rate was compared using Fisher's exact test. In addition, multivariate logistic regression analysis was performed to explore the independent determinants of the success of prognostic prediction.

Results: The success rate of the baPWV articles $(65.7 \%$ [46/70]) tended to be higher than that of the CAVI articles (40.0\% [6/15]; $P=0.083)$. Multivariate analysis demonstrated that $\log$ (number of patients) (OR 11.20, 95\% CI 2.45-51.70, $P=0.002$ ) and dialysis population (OR $0.28,95 \%$ CI $0.08-0.94, P=0.039$ ) were positive and negative independent determinants of the success of prognostic prediction, respectively. In addition, after redefining two studies as the absence of arteriosclerosis obliterans (ASO) exclusion, baPWV (OR 3.36, 95\% CI 0.86-13.20, $P=0.083$ ) and the existence of exclusion criteria of ASO (OR 3.08, 95\% CI 0.96-9.93, $P=0.060$ ) exhibited statistical tendency in the multivariate analysis.

Conclusion: This study demonstrated that the number of study participants and dialysis population were the independent determinants of the success of prognostic prediction. This study also showed the importance of exclusion criteria of ASO when using these indices. In addition, a prospective large-scale study to confirm the superiority in the prognostic prediction of these indices is warranted.

Keywords: peripheral arterial disease, ankle-brachial index, diabetes, hemodialysis, cardiovascular events

\section{Introduction}

The burden of managing atherosclerotic diseases is increasing globally as economic development continues. Pulse wave velocity (PWV) and ankle-brachial index (ABI) have long been used to quantify arteriosclerosis and atherosclerosis, ${ }^{1,2}$ and the clinical significance of each has been established. ${ }^{3-5}$ Carotid-femoral PWV (cfPWV) is a representative marker of arterial stiffness, and several meta-analyses have demonstrated its independent prognostic predictability. ${ }^{6-8}$ Vascular Profiler (VP; BP-203RPE, VP-1000, and VP-2000 series, Japanese product name "form") and VaSera (VS; VS-1000, VS-1500, VS-2000, and VS-3000 series) were first sold in Japan at the end of $1999^{9,10}$ and in the first half of 2002, ${ }^{11,12}$ respectively, which 
were new devices that can simultaneously measure brachial-ankle PWV (baPWV) and ABI. The specifications of VS changed to measure cardio-ankle vascular index (CAVI) and ABI in the first half of 2004, and it was continued to be sold. ${ }^{13,14}$ Several English-language articles about both devices have been published. The first article discussing the prognostic predictability of the baPWV was published in $2005,{ }^{15}$ whereas the first article reporting that of the CAVI was published in 2009. ${ }^{16}$ The number of articles detailing the prognostic predictability of baPWV rapidly increased after 2012, nearing 40 at the end of 2014. ${ }^{17}$ Moreover, three meta-analyses and one rapid communication article using the data derived from one meta-analysis were also published. ${ }^{18-21}$ As information accumulated, the baPWV threshold of $18 \mathrm{~m} / \mathrm{s}$ was set as "high risk,"3,22,23 whereas that of $14 \mathrm{~m} / \mathrm{s}$ was set as "middle risk" 22 in the related guidelines. Five articles reported the prognostic significance of baPWV and CAVI in the same population. ${ }^{24-28}$ However, no studies have comprehensively discussed the differences in prognostic predictability among those indices. ${ }^{29}$ Therefore, this study aimed to identify articles that researched the prognostic predictability of both indices and compare the success rate wholly and in each category. Moreover, this review also aimed to explore the independent predictors of the success of prognostic prediction.

\section{Methods}

\section{Identifying and defining articles}

The concept and measurement method of baPWV and CAVI are available elsewhere. ${ }^{10,14,22}$ The articles identified in this study were written in English (at least in the abstract) and indexed to PubMed or released publicly on the Internet. Each was obtained by the end of April 2018. Figure 1 shows the selection process of the objective studies. The PubMed search was performed using the related keywords such as "pulse wave velocity," "brachial-ankle pulse wave velocity," "cardio-ankle vascular index," "arterial stiffness," and "ankle-brachial index." Longitudinal studies that discussed the prognostic predictability of both indices were identified. End points included all-cause mortality (ACM), cerebrovascular-cardiovascular mortality (CCVM), cerebrovascular-cardiovascular events (CCVE), ischemic heart disease (IHD), major adverse cardiac events, and heart failure. When the other end points such as peripheral arterial disease (PAD) were included, they were explained additionally (Tables 1-3). Studies of functional prognosis such as a decline in cognitive function, kidney function, or activities of daily living were not included. Three meta-analyses and one rapid communication article detailing prognostic predictability of baPWV were excluded. ${ }^{18-21}$ Longitudinal studies that did not include baPWV or CAVI as a prognostic variable were also excluded. ${ }^{30-37}$

Moreover, among the interventional studies in which those indices were measured, those that did not research their prognostic significance were also excluded because of the discrepancy in the research purpose..$^{38,39}$ One study reporting perioperative adverse events was also excluded. ${ }^{40}$ Furthermore, one study that demonstrated the significance of baPWV using a Kaplan-Meier analysis (log-rank test, $P<0.0001)$ was excluded because of considerable difficulty in adopting the Cox proportional hazards model, as the

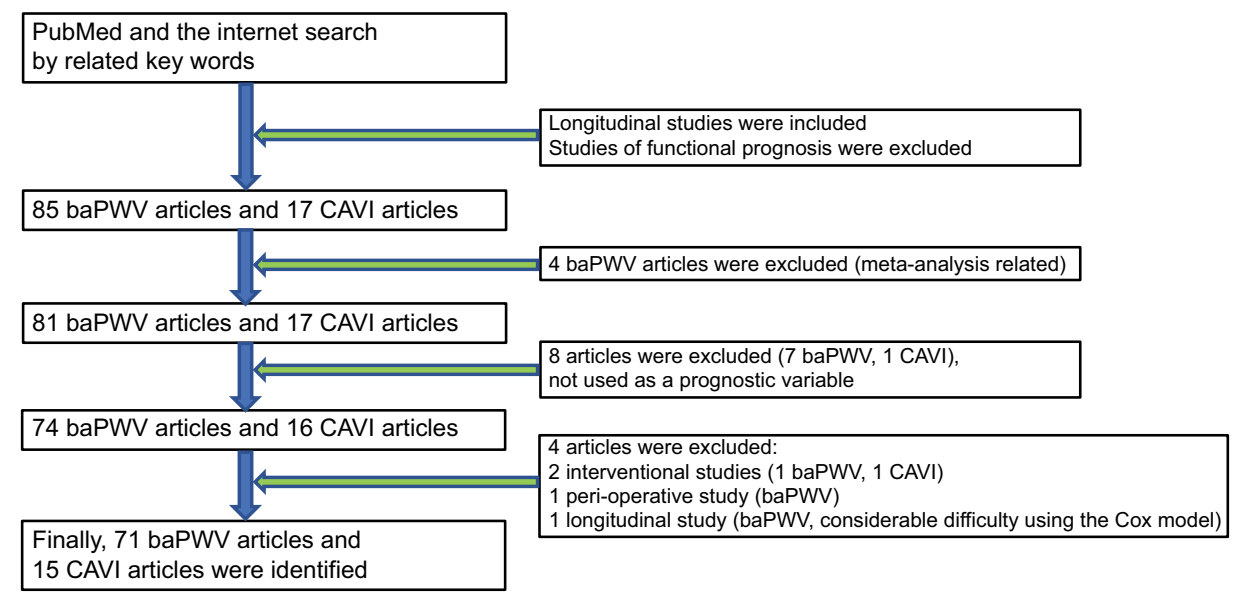

Figure I A flowchart of identifying prognostic studies of baPWV and CAVI articles.

Abbreviations: baPWV, brachial-ankle pulse wave velocity; CAVI, cardio-ankle vascular index. 
Kaplan-Meier curves apparently showed nonproportional changes in the event rates. ${ }^{41}$ Finally, a total of 71 baPWV articles and 15 CAVI articles were identified. The identified studies were categorized according to patient characteristics and the presence or absence of the clarified exclusion criteria of lower extremity (LE)-arteriosclerosis obliterans (ASO)/PAD. Any criteria such as "other vascular diseases" were not defined as the exclusion criteria of LE-ASO/ PAD in this study. The studies demonstrating independent prognostic predictability of those indices on a multivariate Cox proportional hazards model or multivariate logistic regression model were defined as "successful." The studies showing significance on only Kaplan-Meier analysis (log-rank test) and/or not demonstrating statistical significance on a multivariate Cox proportional hazards model were defined as "failed." Comparisons between baPWV and CAVI were performed in all included articles, in the presence or absence of the clarified exclusion criteria of LE-ASO/PAD, in the population of dialysis (hemodialysis and peritoneal dialysis) or other than dialysis, and in the articles discussing both indices in a same cohort. However, one article studying both baPWV and CAVI in the same patient cohort did not describe the prognostic significance of baPWV as a primary end point. As such, that study was excluded from the statistical analysis as a baPWV article because the independent prognostic significance of baPWV for the primary end point was not clarified. ${ }^{28}$ Moreover, because of the relatively ample number of baPWV articles, the studies with or without the clarified exclusion criteria of LE-ASO/PAD only among baPWV articles were compared to confirm the effect on success rate. Furthermore, multivariate logistic regression analysis was performed to explore the independent factors of the success of prognostic prediction.

\section{Statistical analyses}

Statistical analyses were performed using EZR (EZR on $\mathrm{R}$ commander version 1.33 , September 1, 2016). ${ }^{42}$ All comparisons between groups were performed using Fisher's exact test. However, a statistical analysis was not performed among the five articles that simultaneously studied baPWV and CAVI because of the too small sample and of the heterogeneity in the condition. ${ }^{24-28}$ Moreover, logistic regression analysis was performed to explore the independent determinants of the success of prognostic prediction ( success $=1$, failure $=0$ ). The following covariates were analyzed in the univariate analysis: baPWV or CAVI ( $\mathrm{baPWV}=1, \mathrm{CAVI}=0$ ), presence of the clarified exclusion criteria of LE-ASO/PAD (yes $=1$, no $=0$ ), dialysis population (yes $=1$, no $=0$ ), follow-up period (years), age (years), male gender (\%) in the study cohort, and log-transformed number of patients ( $\log \mathrm{NoP})$. The number of patients was $\log$ transformed because of skewed distribution. In this analysis, the mean values of the patients' age and follow-up years were primarily used, and if not available, median values were used. The value of the mean $/$ median patient age was missing in two studies. ${ }^{43,44}$ The proportion of gender was also missing in one study. ${ }^{44}$ Nevertheless, the analysis was performed without these missing data. The covariates whose $P$-value was $\leq 0.2$ in the univariate analysis were entered into the multivariate model. Furthermore, reanalysis was performed by redefining two studies as the absence of LE-ASO/PAD exclusion, ${ }^{27,45}$ because these studies were considered to insufficiently exclude LE-ASO/PAD patients (symptomatic PAD only). $P$-values of $\leq 0.05$ were considered statistically significant, whereas $P$-values of $0.05<P \leq 0.10$ were considered to have a statistical tendency.

\section{Results}

\section{All articles}

A total of 70 articles on baPWV ${ }^{15,24-27,43-107}$ and 15 articles on CAVI ${ }^{16,24-28,108-116}$ were identified. Table 1 presents a summary of these articles. The success rate of independent prognostic predictability of the baPWV articles (65.7\% [46/70]) tended to be higher than that of the CAVI articles $(40.0 \%[6 / 15]$; $P=0.083$; Figure 2).

\section{Articles clarifying the exclusion criteria of LE-ASO/PAD}

Table 2 presents the detailed information about the articles in this category. In this category, the success rates of baPWV and CAVI articles were 75.9\% (22/29) and 57.1\% (4/7), respectively $(P=0.37)$. After excluding two studies that insufficiently excluded LE-ASO/PAD patients, ${ }^{27,45}$ the success rates of baPWV and CAVI articles were $81.5 \%(22 / 27)$ and $66.7 \%(4 / 6)$, respectively $(P=0.58)$.

\section{Articles lacking or not clarifying the exclusion criteria of LE-ASO/PAD}

In this category, the success rates of baPWV articles and CAVI articles were 58.5\% (24/41) and 25\% (2/8), respectively $(P=0.12)$. After adding two studies that insufficiently excluded LE-ASO/PAD patients ${ }^{27,45}$, the success rates of baPWV articles and CAVI articles were 55.8\% (24/43) and $22.2 \%(2 / 9)$, respectively $(P=0.14)$. 


\begin{tabular}{|c|c|c|c|c|c|c|c|c|c|c|c|c|c|c|c|c|}
\hline 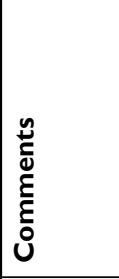 & & 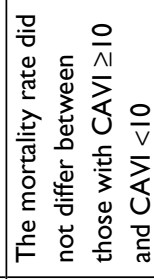 & 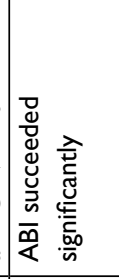 & & 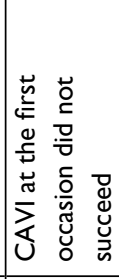 & 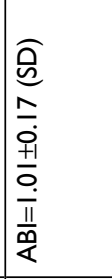 & & & & & & & 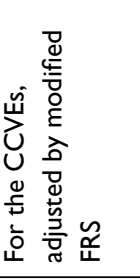 & 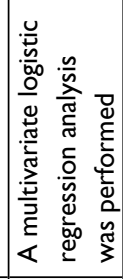 & & 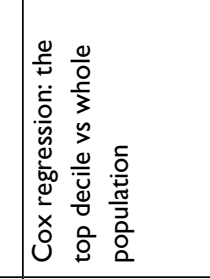 \\
\hline 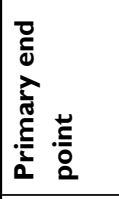 & $\sum_{U}^{\Sigma}$ & 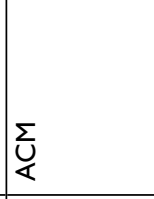 & 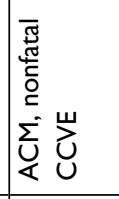 & $\sum_{U}^{\Sigma}$ & यु & \begin{tabular}{|l} 
\\
\\
\end{tabular} & 岂 & & ü & ن) & 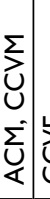 & & 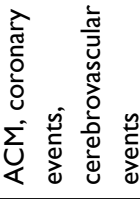 & $\sum_{<}$ & 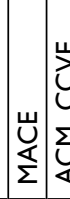 & 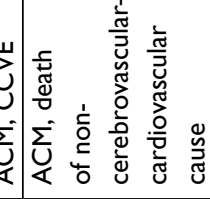 \\
\hline 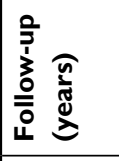 & i & 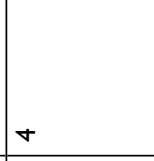 & $\stackrel{m}{m}$ & 艿 & $\stackrel{\text { i }}{\text { in }}$ & $\stackrel{m}{\forall}$ & 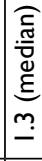 & 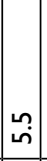 & $\stackrel{\sigma}{\mathbf{i}}$ & & $\stackrel{\infty}{i}$ & & $\stackrel{n}{m}$ & $m$ & సี & 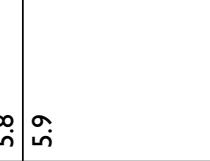 \\
\hline 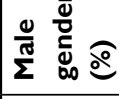 & $\hat{i}$ & $\bar{m}$ & 望 & $\stackrel{t}{0}$ & 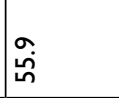 & $\bar{\infty}$ & $\left|\begin{array}{c}\underset{j}{\infty} \\
\infty\end{array}\right|$ & 足: & & 守 & $\begin{array}{l}a \\
\dot{f} \\
\dot{0}\end{array}$ & & مَ & $\stackrel{m}{\hat{0}}$ & స్. & 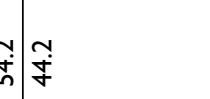 \\
\hline 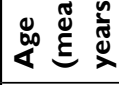 & ต & $\infty$ & రీ & 8 & น & 6 & : & $\left|\begin{array}{l}n \\
\hat{d} \\
m\end{array}\right|$ & & $\overline{0}$ & 今 & 8 & $\bar{\ddots}$ & N & 100 & : \\
\hline 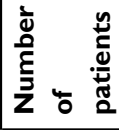 & $\frac{n}{N}$ & $\triangleq$ & 馬 & 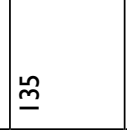 & $\overline{\bar{N}}$ & $\underline{ \pm}$ & $\underset{\sim}{\infty}$ & 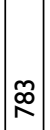 & $\bar{q}$ & $\underline{\underline{n}}$ & $\overline{\widehat{\sigma}}$ & & '. & 号 & 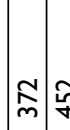 & 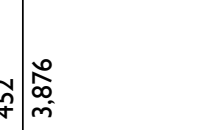 \\
\hline 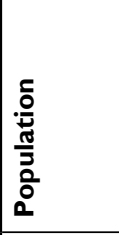 & $\ddot{4}$ & 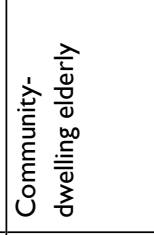 & 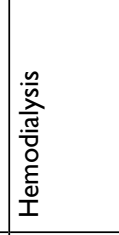 & 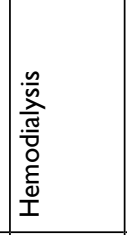 & 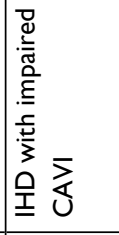 & 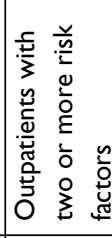 & 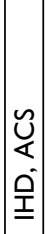 & 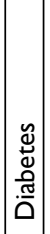 & 울 & 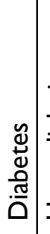 & 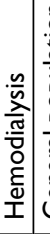 & 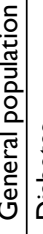 & 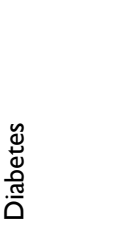 & 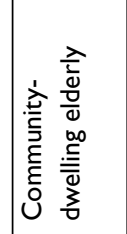 & 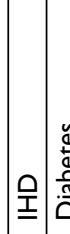 & 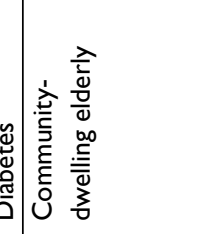 \\
\hline 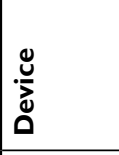 & $\stackrel{9}{>}$ & 5 & 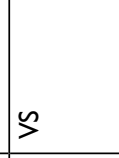 & $s$ & 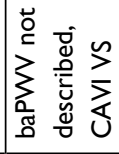 & 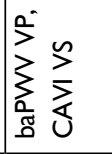 & 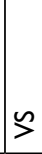 & 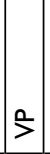 & $\stackrel{9}{>}$ & $\$$ & 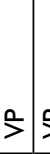 & $s$ & $s$ & $\stackrel{n}{>}$ & $\stackrel{9}{>}$ & 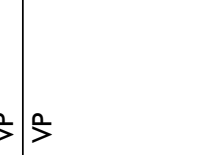 \\
\hline 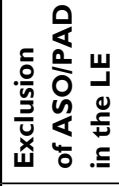 & z & $\stackrel{y}{\succ}$ & ż & $\stackrel{\tilde{\nu}}{\check{\gamma}}$ & $\stackrel{y}{\succ}$ & $\stackrel{\mathscr{e}}{\succ}$ & $\stackrel{\mathscr{y}}{\succ}$ & 苂 & z̀ & $\stackrel{y}{\succ}$ & 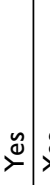 & 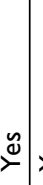 & $\stackrel{\check{\nu}}{\check{\nu}}$ & $\stackrel{y}{\nu}$ & ż & $20 \stackrel{y}{\succ}$ \\
\hline 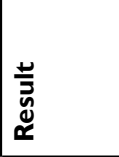 & 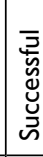 & 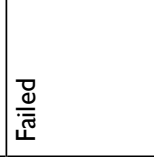 & 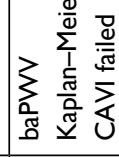 & 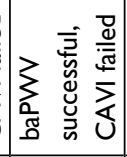 & 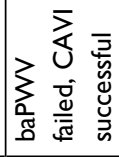 & 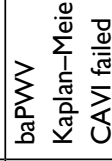 & 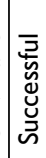 & & 7 & 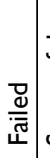 & 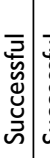 & & 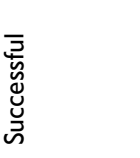 & 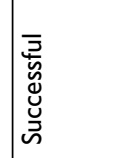 & 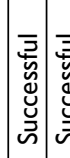 & 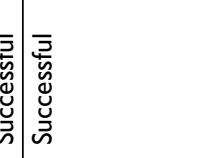 \\
\hline 希 & 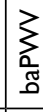 & ¿ & 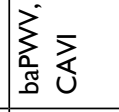 & 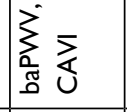 & 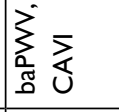 & 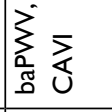 & \্ব & & 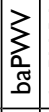 & & & & $\sum_{\substack{0 \\
\text { J }}}$ & $\sum_{\substack{a \\
\text { J } \\
\text { J }}}$ & 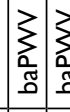 & 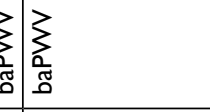 \\
\hline 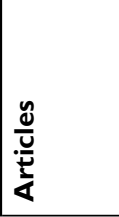 & 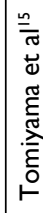 & 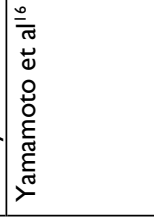 & 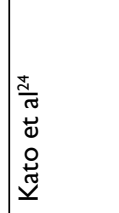 & 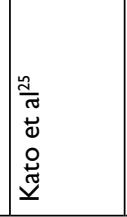 & 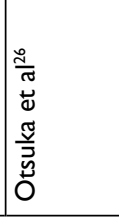 & 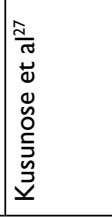 & 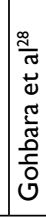 & 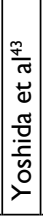 & 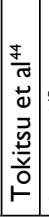 & 部 & 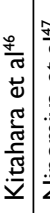 & . & 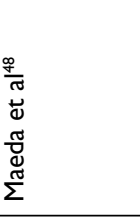 & 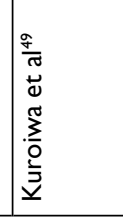 & 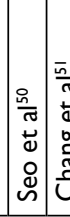 & 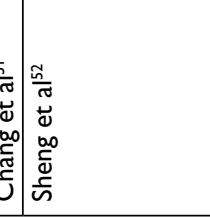 \\
\hline
\end{tabular}




\begin{tabular}{|c|c|c|c|c|c|c|c|c|c|c|c|c|c|c|c|}
\hline 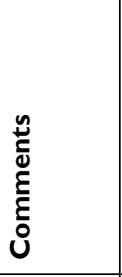 & & & 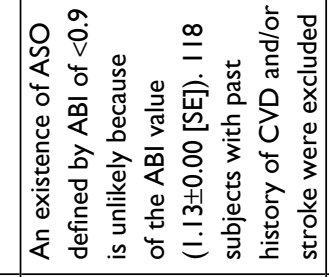 & & & & & 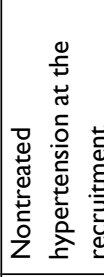 & 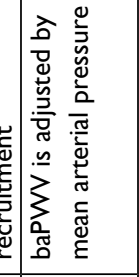 & & & 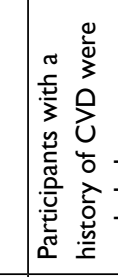 & & & \\
\hline 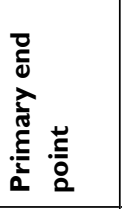 & 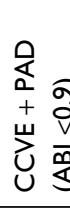 & 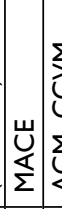 & & |ü| & ư & & & ü & 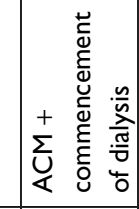 & 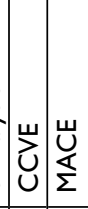 & & 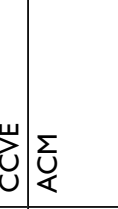 & 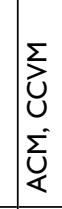 & 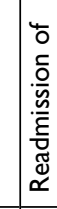 & 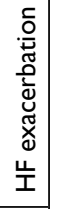 \\
\hline 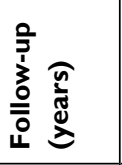 & 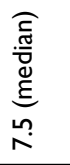 & $\mid$ & & 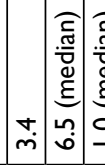 & : & m. & $\cong$ & $m$ & $\cong$ & 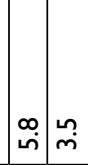 & & $\overline{\mathrm{v}}$ & $m$ & $\cong$ & \\
\hline 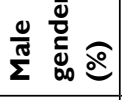 & ن. & 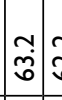 & y. & 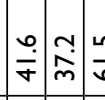 & $\begin{array}{l}: \\
0\end{array}$ & 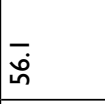 & & 字 & $\mid \begin{array}{c}m \\
\infty \\
0\end{array}$ & 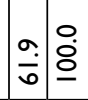 & & $\begin{array}{c}\dot{\infty}_{\infty}^{\infty} \\
\end{array}$ & $\bar{m}$ & 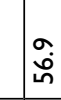 & \\
\hline 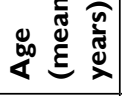 & in & in & 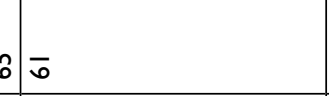 & $\pi \%$ & f) & $\overline{0}$ & & 8 & 8 & $\because \%$ & & 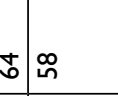 & 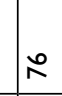 & 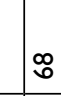 & \\
\hline 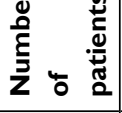 & : & 忞 & & $\mid$ & $=\underset{\alpha}{\infty}$ & q & $\stackrel{\varpi}{\varrho}$ & ర్రి & 占 & ఏ & & 岕 & 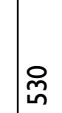 & $\operatorname{s}$ & \\
\hline 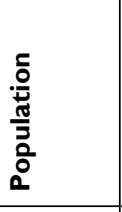 & 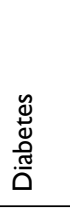 & 呈 & 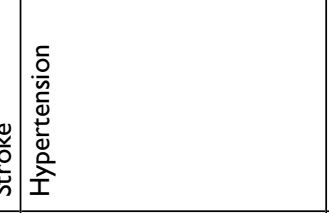 & 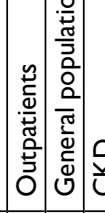 & 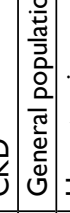 & & & 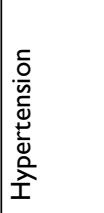 & Uे & 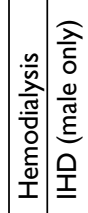 & & 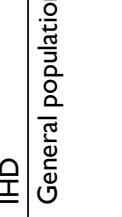 & & 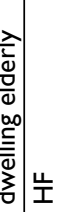 & \\
\hline هัّ & $>$ & $\stackrel{s}{>}$ & $\stackrel{2}{>}$ & $\$ \$$ & $>$ & 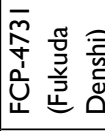 & $>$ & $p$ & 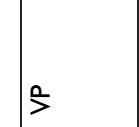 & $>>$ & & $\$$ & $p$ & $\stackrel{9}{>}$ & \\
\hline 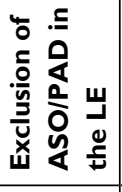 & $\stackrel{\mathscr{y}}{\succ}$ & 20 & 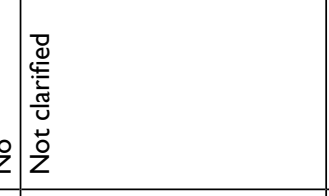 & 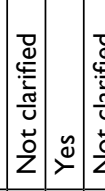 & & $\stackrel{y}{\nu}$ & 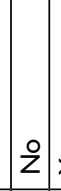 & $\stackrel{y}{\nu}$ & 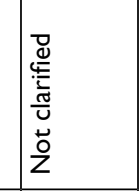 & 它 & & 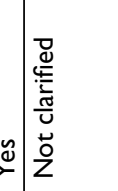 & ֻே & $\stackrel{y}{>}$ & \\
\hline 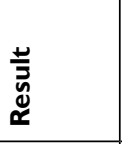 & 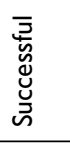 & 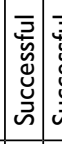 & 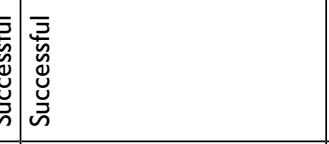 & 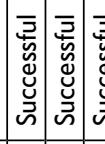 & & & $\mid$ & | & 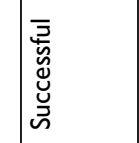 & 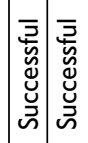 & & 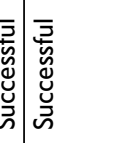 & $\mid$ & 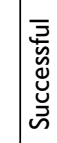 & \\
\hline 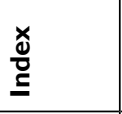 & 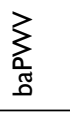 & 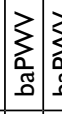 & 岁 & 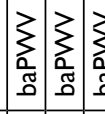 & $\left|\sum_{\substack{0 \\
0}}\right|$ & $\sum_{\substack{0 \\
\text { J }}}^{3}$ & & $\sum_{\substack{0 \\
\text { J }}}^{3}$ & $\sum_{\substack{0 \\
\text { J }}}^{3}$ & 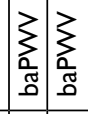 & . & 党 & $\sum_{\substack{0 \\
\text { d. }}}^{3}$ & 经 & \\
\hline $\begin{array}{l}\frac{0}{4} \\
\frac{\sin }{4}\end{array}$ & 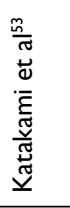 & 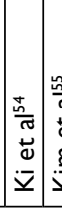 & 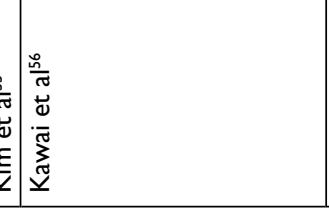 & 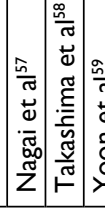 & & & $\mid$ & 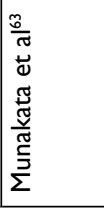 & 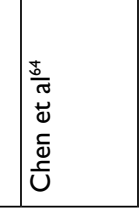 & 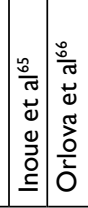 & & 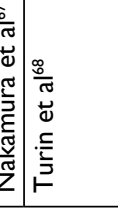 & 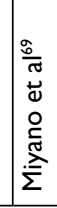 & 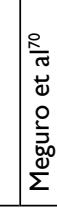 & \\
\hline
\end{tabular}




\begin{tabular}{|c|c|c|c|c|c|c|c|c|c|c|c|c|c|c|c|c|c|c|c|c|c|c|c|c|c|c|}
\hline 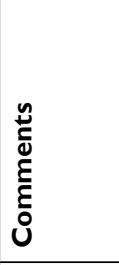 & & & & & & & & & & 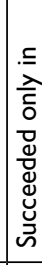 & & & & & & & & & & & & & & & 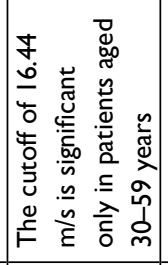 & \\
\hline 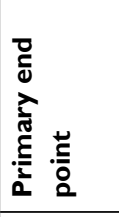 & $\sum_{U}^{\Sigma}$ & 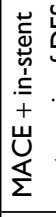 & & ư & $\begin{array}{l}\Sigma \\
\mathbf{U} \\
\end{array}$ & 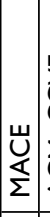 & 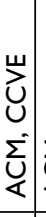 & & 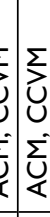 & \begin{tabular}{|l} 
\\
$\frac{u}{\Sigma}$ \\
$\Sigma$
\end{tabular} & & u & 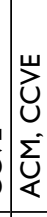 & $\begin{array}{l}\mathrm{U} \\
\mathbf{u} \\
\Sigma \\
\end{array}$ & $\begin{array}{l}\Sigma \\
\bar{Q} \\
\end{array}$ & \begin{tabular}{|l} 
\\
$\bar{\Sigma}$ \\
$\Sigma$ \\
\end{tabular} & $\begin{array}{l}0 \\
\vdots \\
\vdots\end{array}$ & & 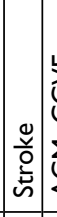 & 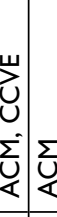 & 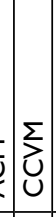 & 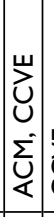 & Ü & 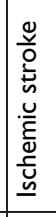 & $\begin{array}{l}\text { Uu } \\
\Sigma \\
\Sigma\end{array}$ & 㞬 \\
\hline 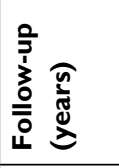 & $\stackrel{\nabla}{m}$ & $\bar{i}$ & 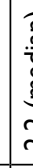 & 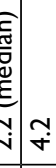 & in & $\begin{array}{c}n \\
m\end{array} \mid$ & . & +5 & $\dot{v} \underset{j}{j}$ & $\stackrel{\infty}{0}$ & & $\underbrace{\infty}_{-\infty}$ & $\underbrace{+}_{\dot{*}}$ & 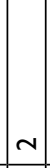 & 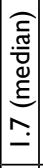 & $\begin{array}{c}m \\
\text { in }\end{array}$ & 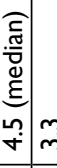 & & $\begin{array}{l}a \\
\end{array}$ & $\stackrel{\Delta}{*}$ & 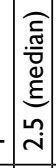 & in. & 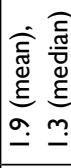 & 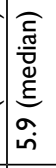 & in & $f$ \\
\hline 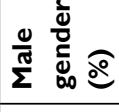 & 筡 & ஸे & ga & $\begin{array}{l}0_{0}^{0} \\
0 \\
0\end{array}$ & $\frac{0}{5}$ & 음 & م. & $\frac{\infty}{0}$ & $\begin{array}{l}0 \\
\dot{f}\end{array} \mid \begin{array}{c}m \\
0 \\
0\end{array}$ & $\stackrel{\text { Iீ}}{i}$ & & $\dot{\stackrel{j}{j}}$ & fo & $\begin{array}{l}0 \\
\dot{0} \\
\tilde{0}\end{array}$ & I & $\frac{\text { ne? }}{i}$ & $\begin{array}{l}0 \\
\dot{j} \\
j_{1}\end{array}$ & & $\left.\begin{array}{l}r \\
\tilde{n}\end{array}\right]$ & $\begin{array}{l}m \\
\dot{f}\end{array}$ & 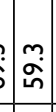 & $\frac{9}{n}$ & $\overline{\hat{\sigma}}$ & ì. & $\stackrel{2}{R}$ & 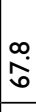 \\
\hline
\end{tabular}

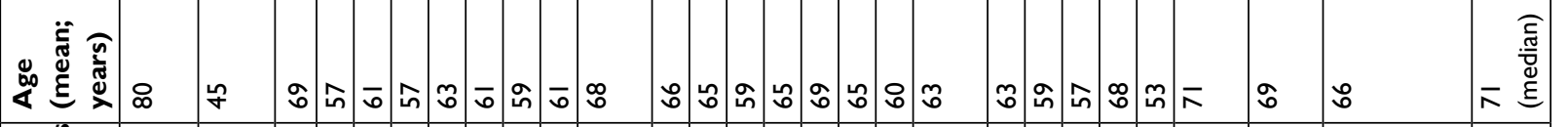

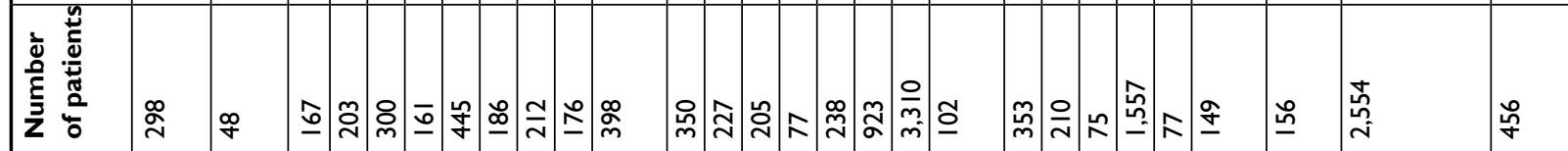

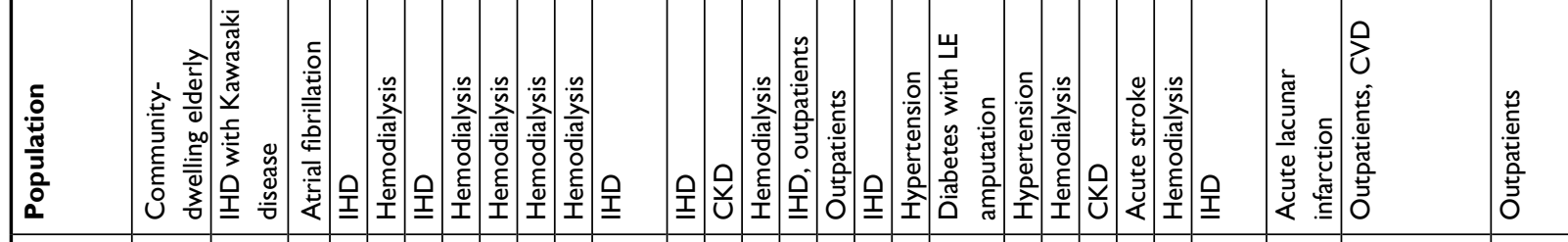

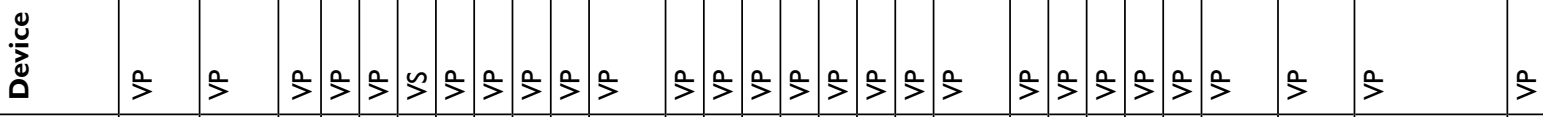

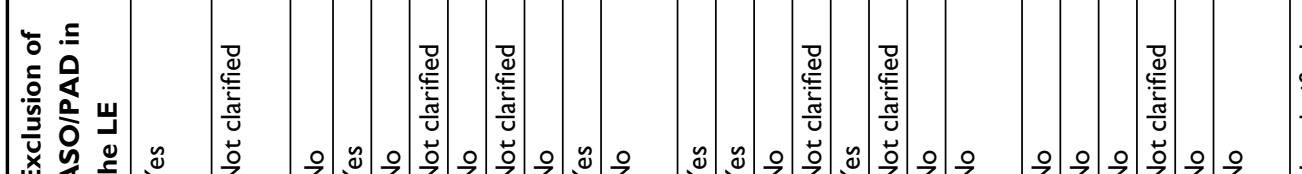

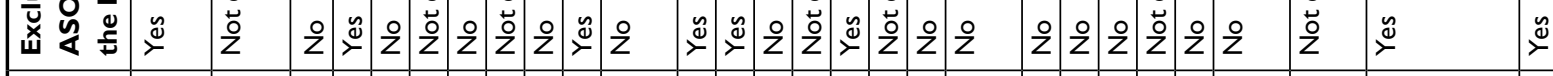

\begin{tabular}{|c|c|c|c|c|c|c|c|c|c|c|c|c|c|c|c|c|c|c|c|c|c|}
\hline 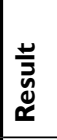 & \begin{tabular}{|l}
$\bar{\Xi}$ \\
$\overline{\breve{y}}$ \\
$\breve{u ̈}$ \\
$心$ \\
$心$
\end{tabular} & 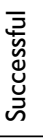 & 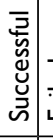 & 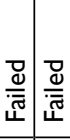 & 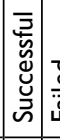 & & 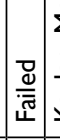 & 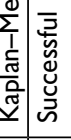 & $\mid$ & & 0 & & & $\begin{array}{l}\bar{\Xi} \\
\overline{\breve{y}} \\
\breve{u ̈ ~} \\
心\end{array}$ & 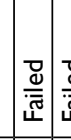 & & 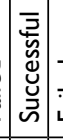 & 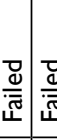 & 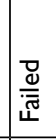 & 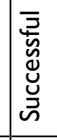 & \\
\hline 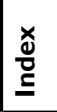 & $\sum_{\substack{0 \\
\text { J }}}^{3}$ & 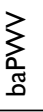 & 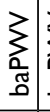 & 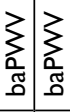 & 﨎 & & 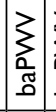 & 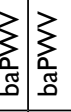 & $\sum_{\substack{0 \\
\tilde{J}}}^{3}$ & & $\sum_{\substack{0 \\
\tilde{J}}}$ & 13 & & $\sum_{\substack{0 \\
\text { J }}}^{3}$ & $\sum_{\substack{n \\
\tilde{J}}}$ & 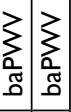 & 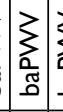 & 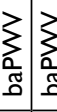 & $\sum_{\substack{a \\
\text { a }}}^{3}$ & $\sum_{\substack{a \\
\text { I. }}}^{3}$ & $\frac{\pi}{\pi}$ \\
\hline$\frac{0}{\mathscr{N}}$ & 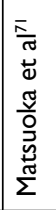 & 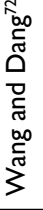 & 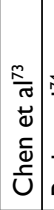 & 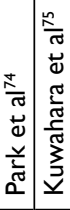 & 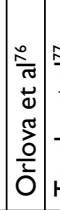 & 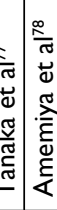 & 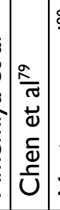 & 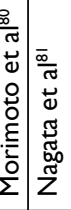 & 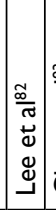 & 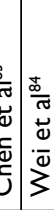 & $\begin{array}{l}0 \\
0 \\
0 \\
\underline{\underline{0}}\end{array}$ & $\frac{.}{\sigma}$ & & 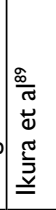 & 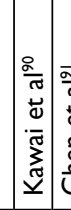 & 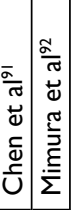 & 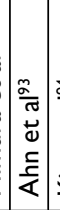 & 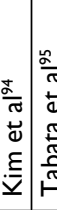 & 总 & 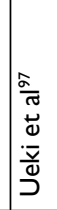 & 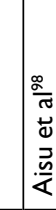 \\
\hline
\end{tabular}




\begin{tabular}{|c|c|c|c|c|c|c|c|c|c|c|c|c|c|c|}
\hline 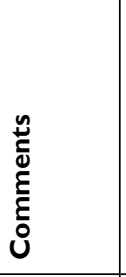 & & & & & 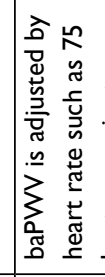 & & & & 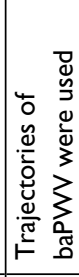 & & & 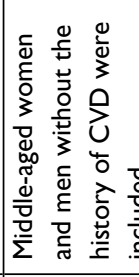 & 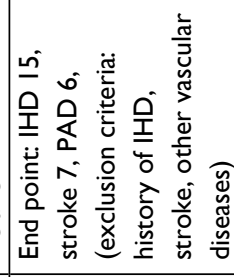 & \\
\hline 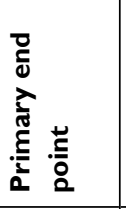 & 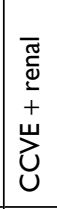 & & 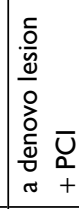 & ü & ü & ü & & 己े & & 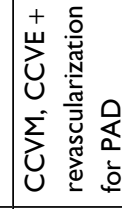 & 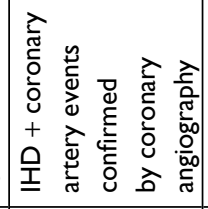 & & $\begin{array}{l}0 \\
0 \\
0 \\
+ \\
\breve{u} \\
己 \\
u\end{array}$ & ü \\
\hline 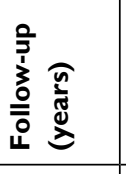 & m & $n$ & $\Xi$ & - & 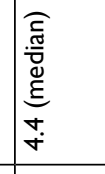 & $\stackrel{\infty}{\sim}$ & & ن. & & 字 & $\hat{\omega}$ & $\stackrel{\infty}{m}$ & n & $\underset{\dot{r}}{\stackrel{m}{*}}$ \\
\hline 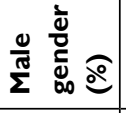 & $\begin{array}{l} \\
\\
\end{array}$ & $\mid \begin{array}{c}0 \\
\substack{\infty \\
n}\end{array}$ & $\hat{\omega}$ & 於 & 㐯 & 尊 & & : & $\stackrel{0}{\dot{y}}$ & 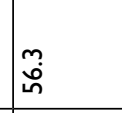 & $\frac{1}{\min }$ & 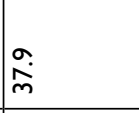 & 朵 & 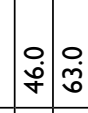 \\
\hline 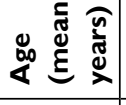 & (2 & $\infty$ & in & t & ก & $\pi$ & & ס. & $\pi$ & ๓0 & 0 & H' & f & ¿ \\
\hline 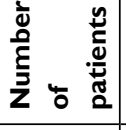 & 守 & ले & $\bar{\sigma}$ & $\overline{\bar{\sigma}}$ & $\mid \begin{array}{l}\bar{a} \\
\bar{y} \\
\end{array}$ & శั & & બે & $\underset{=}{ \pm}$ & $\stackrel{\text { i }}{i}$ & $\tilde{o}_{-}^{\circ}$ & $\frac{10}{4}$ & 요 & 必 \\
\hline 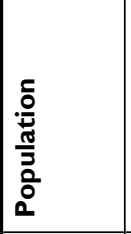 & U & & 올 & 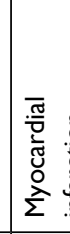 & 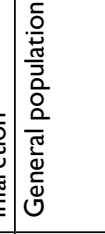 & 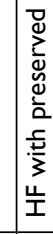 & & 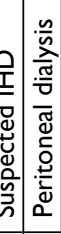 & 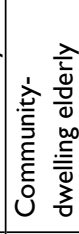 & 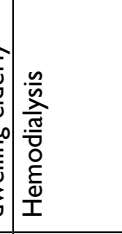 & 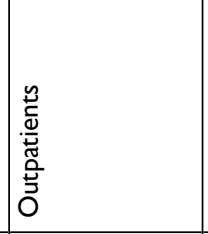 & 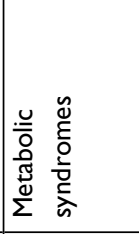 & 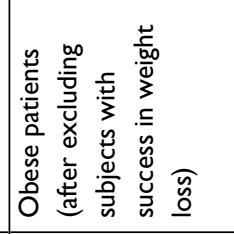 & 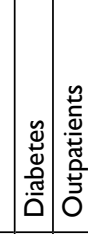 \\
\hline هัّ & 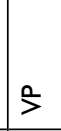 & $\stackrel{s}{>}$ & $>$ & $\stackrel{\rho}{>}$ & $\stackrel{p}{>}$ & $\rho$ & 12 & 9 & $p$ & 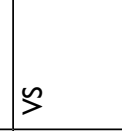 & 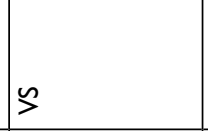 & $s$ & $s$ & $>>$ \\
\hline 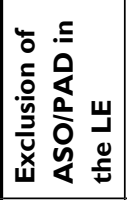 & 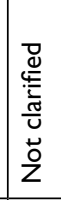 & $\stackrel{0}{z}$ & z & $\stackrel{\circ}{z}$ & $\stackrel{\check{z}}{\rightleftharpoons}$ & $\stackrel{\ddot{\Delta}}{\tau}$ & & 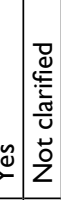 & 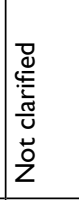 & ż & $\stackrel{y}{x}$ & 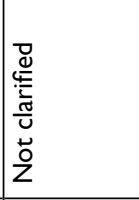 & 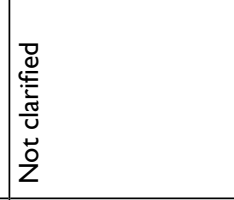 & 은 \\
\hline 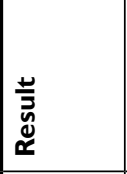 & 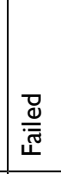 & 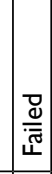 & $\frac{\bar{v}}{\frac{\vec{v}}{\bar{w}}}$ & 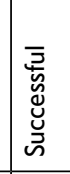 & 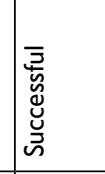 & 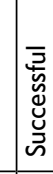 & & & & $\frac{\widetilde{z}}{\frac{\tilde{w}}{\tilde{w}}}$ & 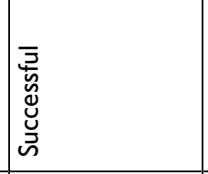 & 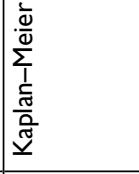 & \begin{tabular}{|l}
$\bar{z}$ \\
总 \\
$\bar{n}$ \\
\end{tabular} & 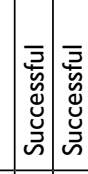 \\
\hline 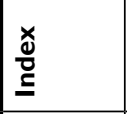 & 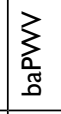 & 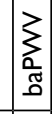 & $\sum_{\substack{0 \\
\text { d }}}^{3}$ & $\sum_{\substack{a \\
\text { J }}}^{3}$ & 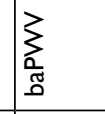 & 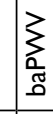 & & כ) & $\begin{array}{l}3 \\
\text { and } \\
\text { J } \\
\end{array}$ & 式 & 矛 & 永 & 文 & 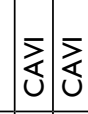 \\
\hline 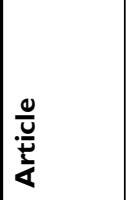 & 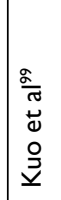 & 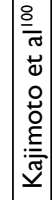 & 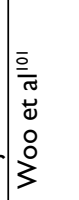 & 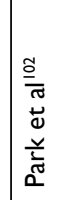 & 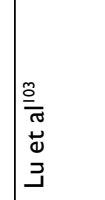 & 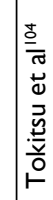 & & 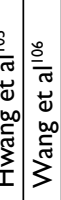 & 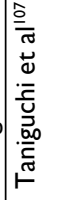 & 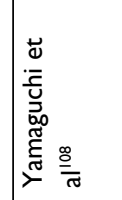 & 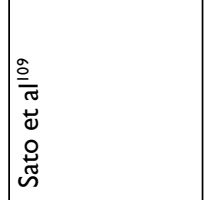 & 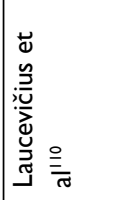 & 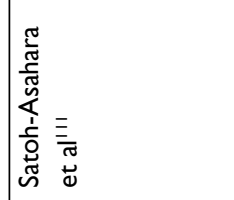 & 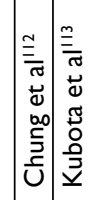 \\
\hline
\end{tabular}



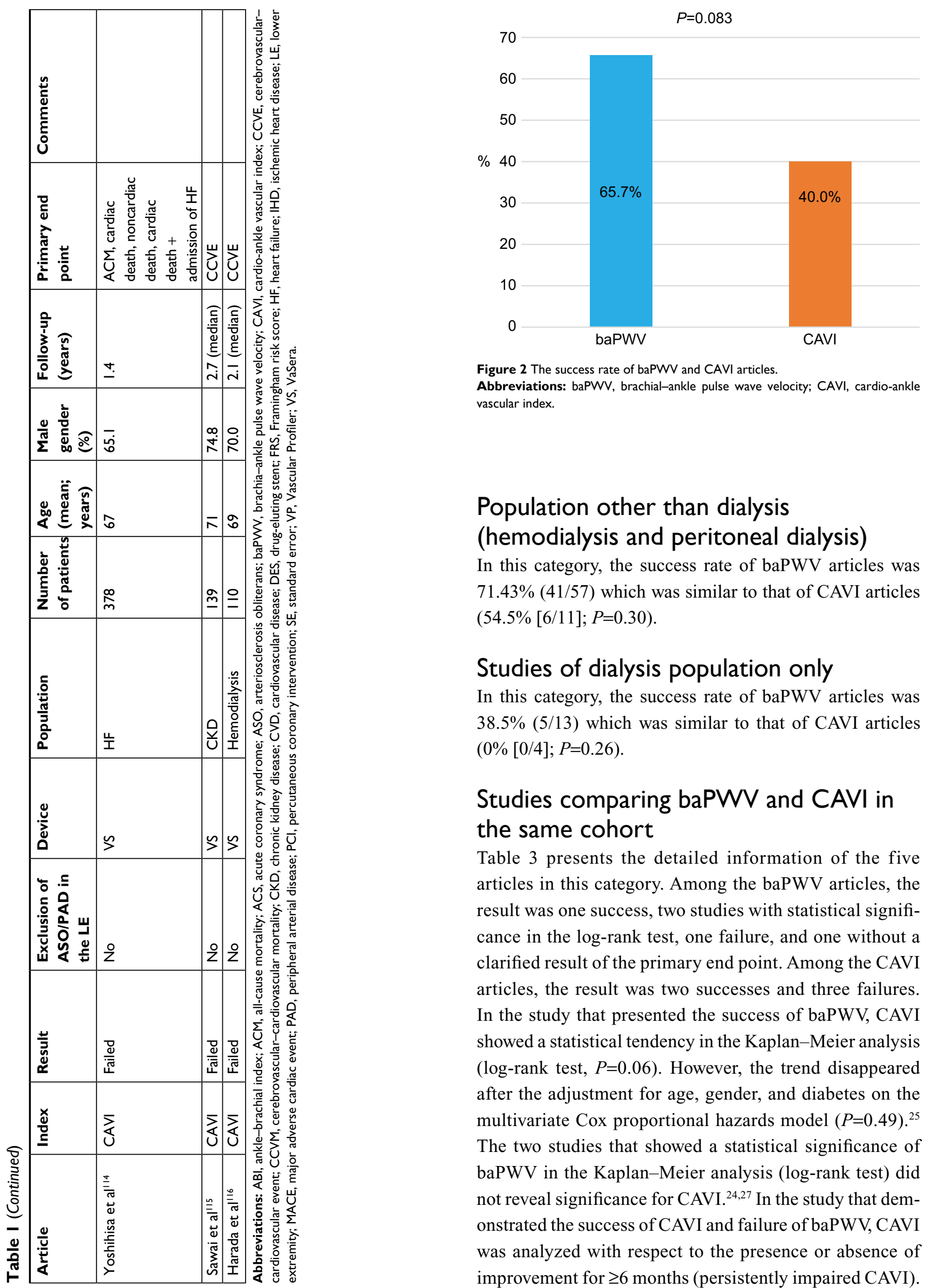

Figure 2 The success rate of baPWV and CAVI articles.

Abbreviations: baPWV, brachial-ankle pulse wave velocity; CAVI, cardio-ankle vascular index.

\section{Population other than dialysis (hemodialysis and peritoneal dialysis)}

In this category, the success rate of baPWV articles was $71.43 \%$ (41/57) which was similar to that of CAVI articles (54.5\% [6/11]; $P=0.30)$.

\section{Studies of dialysis population only}

In this category, the success rate of baPWV articles was $38.5 \%(5 / 13)$ which was similar to that of CAVI articles $(0 \%[0 / 4] ; P=0.26)$.

\section{Studies comparing baPWV and CAVI in the same cohort}

Table 3 presents the detailed information of the five articles in this category. Among the baPWV articles, the result was one success, two studies with statistical significance in the log-rank test, one failure, and one without a clarified result of the primary end point. Among the CAVI articles, the result was two successes and three failures. In the study that presented the success of baPWV, CAVI showed a statistical tendency in the Kaplan-Meier analysis (log-rank test, $P=0.06$ ). However, the trend disappeared after the adjustment for age, gender, and diabetes on the multivariate Cox proportional hazards model $(P=0.49) .{ }^{25}$ The two studies that showed a statistical significance of baPWV in the Kaplan-Meier analysis (log-rank test) did not reveal significance for CAVI. ${ }^{24,27}$ In the study that demonstrated the success of CAVI and failure of baPWV, CAVI was analyzed with respect to the presence or absence of improvement for $\geq 6$ months (persistently impaired CAVI). 
However, the baPWV's raw value at the second occasion of the measurement was analyzed. ${ }^{26}$ In a study in which the baPWV result was defined as "not clarified," the prognostic significance of baPWV in the multivariate Cox proportional hazards model analysis on the primary end point was not described. However, the prognostic significance of CAVI (not baPWV) on the secondary end point of nonfatal stroke was presented. ${ }^{28}$

\section{Comparison of baPWV articles according to the presence or absence of the clarified exclusion criteria of LE-ASO/ PAD}

The success rate of the articles in the presence of these criteria $(75.9 \%$ [22/29]) was similar to that in their absence (58.5\% [24/41]; $P=0.20$; Figure 3A). However, after redefining two studies as the absence of LE-ASO/PAD exclusion, ${ }^{27,45}$ the success rate of the articles in the presence of these criteria (81.5\% [22/27]) was significantly higher than that of the articles in the absence (55.8\% [24/43]; $P=0.039$; Figure 3B).

\section{Multivariate logistic regression analysis to identify the independent determinants for the success of prognostic prediction}

Table 4 presents this result. In the univariate analysis, $\log \operatorname{NoP}(P=0.0006)$ and dialysis population $(P=0.005)$ were significantly associated with the success of prognostic prediction, whereas baPWV and exclusion of LE-ASO/PAD showed a statistical tendency $(P=0.071, P=0.076$, respectively). In the multivariate analysis, $\log$ NoP (OR 11.20, 95\% CI 2.45-51.70, $P=0.002$ ) and dialysis population (OR 0.28, 95\% CI 0.08-0.94, $P=0.039$ ) were identified as the independent determinants of the success of prognostic prediction.

Table 5 summarizes the result after redefining two studies as the absence of LE-ASO/PAD exclusion. ${ }^{27,45}$ In the univariate analysis, exclusion of LE-ASO/PAD $(P=0.01)$, dialysis population $(P=0.005)$, and $\log \operatorname{NoP}(P=0.0006)$ were significantly associated with the success of prognostic prediction, whereas baPWV showed statistical tendency $(P=0.071)$. In the multivariate analysis, $\log \mathrm{NoP}$ and dialysis population were the statistically significant factors (for log NoP: OR 9.04, 95\% CI 1.90-43.00, $P=0.006$; for dialysis population: OR $0.27,95 \%$ CI $0.07-0.96, P=0.043$ ). However, baPWV and the exclusion of LE-ASO/PAD showed statistical tendency in the success of prognostic prediction (for baPWV: OR 3.36,
95\% CI 0.86-13.20, $P=0.083$; for the exclusion of LE-ASO/ PAD: OR 3.08, 95\% CI 0.96-9.93, $P=0.060$ ).

\section{Discussion Overview}

To the best of our knowledge, this study is the first to comprehensively review the prognostic predictability of baPWV and CAVI. The current number of English articles using the indices of VP and VS is approximately 1,800 and 550, respectively. ${ }^{117}$ The ratio of articles studying the prognostic significance of these parameters did not differ significantly (baPWV 70/1,800; CAVI 15/550, $P=0.24$ ). There were five articles that simultaneously studied prognostic significance of baPWV and CAVI in a same patient population. The success rate of baPWV articles tended to be higher than that of CAVI articles (65.7\% vs $40.0 \%, P=0.083)$. Dialysis population and $\log$ NoP were the independent determinants for the success in the multivariate logistic regression analysis (Table 4). Moreover, after redefining two studies as the absence of exclusion of LE-ASO/PAD, ${ }^{27,45}$ the success rate in the presence of these criteria was significantly higher than that in the absence of these criteria among the baPWV articles only ( $81.5 \%$ vs $55.8 \%, P=0.039)$. Furthermore, multivariate logistic analysis showed that baPWV and exclusion of LE-ASO/PAD had a statistical trend on the success of prognostic prediction ( $P=0.083, P=0.060$, respectively). The multivariate analysis also showed that the effects of dialysis population and $\log \mathrm{NoP}$ were attenuated, although these parameters were still significant $(P=0.043, P=0.006$, respectively; Table 5). Therefore, $\log$ NoP had the strongest power on the success of prognostic prediction among the articles investigated in this study. Actually, the most studies involving $>1,000$ participants were successful in the prognostic prediction. ${ }^{47,48,52,53,55,58,68,88,93,97,103,107,109}$ These studies mostly used baPWV, and one study used CAVI. ${ }^{109}$ However, the largest study of CAVI involving $>2,000$ participants failed. ${ }^{110}$ Moreover, this review confirmed that the dialysis population (mostly hemodialysis) was a negative determinant of the success of prognostic prediction of the baPWV and CAVI. This is not surprising because the incidence of having false-negative LE-ASO/PAD is high even if the exclusion criteria are defined as ABI of $\leq 0.9$ in the hemodialysis population. Age, proportion of male gender, and follow-up period in the study population had no effect on the success of baPWV and CAVI in the prognostic prediction. In general, age and gender affect 
Table 2 Detailed information about baPWV and CAVI articles in which ASO and/or PAD was excluded from the analysis

\begin{tabular}{|c|c|c|c|c|c|c|c|c|}
\hline Articles & Index & Result & $\begin{array}{l}\text { Criteria of } \\
\text { ASO/PAD } \\
\text { exclusion }\end{array}$ & Device & Population & $\begin{array}{l}\text { Number } \\
\text { of } \\
\text { patients }\end{array}$ & $\begin{array}{l}\text { Age } \\
\text { (mean; } \\
\text { years) }\end{array}$ & $\begin{array}{l}\text { Male } \\
\text { gender } \\
(\%)\end{array}$ \\
\hline Yamamoto et al ${ }^{16}$ & CAVI & Failed & ASO & VS & $\begin{array}{l}\text { Community- } \\
\text { dwelling } \\
\text { elderly }\end{array}$ & 117 & 80 & 31.1 \\
\hline Kato et $\mathrm{al}^{25}$ & baPWV & Successful & $\mathrm{ABI}<0.9$ & VS & Hemodialysis & 135 & 60 & 67.4 \\
\hline Kato et $\mathrm{al}^{25}$ & CAVI & Failed & $\mathrm{ABI}<0.9$ & VS & Hemodialysis & 135 & 60 & 67.4 \\
\hline Otsuka et al ${ }^{26}$ & baPWV & Failed & PAD & $\begin{array}{l}\text { Not } \\
\text { described }\end{array}$ & $\begin{array}{l}\text { IHD with } \\
\text { impaired } \\
\text { CAVI }\end{array}$ & 211 & 65 & 55.9 \\
\hline Otsuka et al ${ }^{26}$ & CAVI & Successful & PAD & VS & $\begin{array}{l}\text { IHD with } \\
\text { impaired } \\
\text { CAVI }\end{array}$ & 211 & 65 & 55.9 \\
\hline Kusunose et $\mathrm{al}^{27}$ & baPWV & Failed & Symptomatic PAD & VP & $\begin{array}{l}\text { Outpatients } \\
\text { with two or } \\
\text { more risk } \\
\text { factors }\end{array}$ & 114 & 69 & 78.1 \\
\hline Kusunose et $\mathrm{a}^{27}$ & CAVI & Failed & Symptomatic PAD & VS & $\begin{array}{l}\text { Outpatients } \\
\text { with two or } \\
\text { more risk } \\
\text { factors }\end{array}$ & 114 & 69 & 78.1 \\
\hline Gohbara et al ${ }^{28}$ & CAVI & Successful & PAD & VS & IHD, ACS & 288 & 65 & 82.3 \\
\hline Lau et $\mathrm{al}^{45}$ & baPWV & Failed & Symptomatic PAD & VP & Diabetes & $15 \mid$ & 61 & 40.4 \\
\hline Kitahara et $\mathrm{al}^{46}$ & baPWV & Successful & $\mathrm{ABI}<0.9$ & VP & Hemodialysis & 671 & 59 & 64.9 \\
\hline Ninomiya et al ${ }^{47}$ & baPWV & Successful & $\mathrm{ABI}<0.9$ & VP & $\begin{array}{l}\text { General } \\
\text { population }\end{array}$ & 2,916 & 60 & 42.7 \\
\hline Maeda et $\mathrm{al}^{48}$ & baPWV & Successful & $\mathrm{ABI}<0.9$ & $\mathrm{VP}$ & Diabetes & 3,628 & 61 & 59.7 \\
\hline
\end{tabular}




\begin{tabular}{|c|c|c|c|c|c|c|}
\hline $\begin{array}{l}\text { Follow- } \\
\text { up } \\
\text { (years) }\end{array}$ & $\begin{array}{l}\text { Primary end } \\
\text { point }\end{array}$ & $\begin{array}{l}\text { Number } \\
\text { of } \\
\text { events }\end{array}$ & Cutoff & HR (adjusted) & $\begin{array}{l}\text { HR as a } \\
\text { continuous } \\
\text { variable (adjusted) }\end{array}$ & Comments \\
\hline 4 & ACM & 14 & 10 & Not provided & Not provided & $\begin{array}{l}\text { The mortality rate did } \\
\text { not differ between } \\
\text { patients with } C A V I \\
\geq \mid 0 \text { and } C A V \mid<10\end{array}$ \\
\hline 5.3 & CCVM & 22 & $\begin{array}{l}16.6 \mathrm{~m} / \mathrm{s} \text { (the } \\
\text { top tertile) }\end{array}$ & $\begin{array}{l}16.9(95 \% \mathrm{Cl} \\
\mathrm{I} . \mathrm{I}-25 \mathrm{I} .8, P=0.04)\end{array}$ & Not provided & \\
\hline 5.3 & CCVM & 22 & $\begin{array}{l}9.9 \text { (the top } \\
\text { tertile) }\end{array}$ & Failed & Not provided & \\
\hline 2.9 & CCVE & 28 & $\begin{array}{l}\text { baPWV at } \\
\text { the second } \\
\text { occasion }\end{array}$ & Failed & Not provided & \\
\hline 2.9 & CCVE & 28 & $\begin{array}{l}\text { Persistently } \\
\text { impaired } \\
\text { CAVI in } 6 \\
\text { months }\end{array}$ & $\begin{array}{l}\text { Persistently } \\
\text { impaired CAVI: } 3.3 \\
(95 \% \mathrm{Cl} \text { I.47-8.59, } \\
P<0.0 \mathrm{I})\end{array}$ & Not provided & $\begin{array}{l}\text { CAVI at the first } \\
\text { occasion did not } \\
\text { succeed }\end{array}$ \\
\hline 4.3 & MACE & 35 & $17.03 \mathrm{~m} / \mathrm{s}$ & Not provided & Failed & $\mathrm{ABI}=\mathrm{I} .0 \mathrm{I} \pm 0 . \mathrm{I}(\mathrm{SD})$ \\
\hline 4.3 & MACE & 35 & 9.2 & Not provided & Failed & $A B I=1.01 \pm 0.17(S D)$ \\
\hline $\begin{array}{l}\text { I.3 } \\
\text { (median) }\end{array}$ & CCVE & 19 & 8.325 & $\begin{array}{l}18(95 \% \mathrm{Cl} 2.369- \\
136.8, P=0.005)\end{array}$ & Not provided & $\begin{array}{l}\text { In the secondary } \\
\text { end point (nonfatal } \\
\text { ischemic stroke), CAVI } \\
\text { succeeded, baPWV } \\
\text { failed }\end{array}$ \\
\hline 5.1 & CCVE & 16 & $14.67 \mathrm{~m} / \mathrm{s}$ & Not provided & Failed & \\
\hline 2.8 & ACM, CCVM & 86,55 & $\begin{array}{l}19.6 \mathrm{~m} / \mathrm{s} \\
\text { (the third } \\
\text { quartile), } 23 \\
\mathrm{~m} / \mathrm{s} \text { (the top } \\
\text { quartile) }\end{array}$ & $\begin{array}{l}\text { ACM, the third } \\
\text { quartile: } 3.32 \text { ( } 95 \% \\
\text { Cl I.22-9.02, } \\
P=0.019) \text {, the } \\
\text { top quartile: } 4.08 \\
(95 \% \mathrm{Cl} \text { I.46- } \\
\text { II.43, } P=0.007) / \\
\text { cardiovascular } \\
\text { mortality, the top } \\
\text { quartile: } 7.03 \text { ( } 95 \% \\
C I \quad 1.49-33.08, \\
P=0.014)\end{array}$ & Not provided & \\
\hline 7.1 & CCVE & 126 & $17.6 \mathrm{~m} / \mathrm{s}$ & $\begin{array}{l}\text { The third quintile: } \\
7.17 \text { (95\% Cl I.66- } \\
3 \mathrm{I} .03, P=0.008) \text {, the } \\
\text { fourth quintile: } 8.77 \\
\text { (95\% CI I.99-38.7I, } \\
P=0.004) \text {, the top } \\
\text { quintile: I2.20 (95\% } \\
\mathrm{Cl} 2.68-55.64 \text {, } \\
P=0.00 \mathrm{I})\end{array}$ & $\begin{array}{l}\text { Per } 20 \% \text { of baPWV, } \\
\text { I.3I }(95 \% \mathrm{Cl} \\
\mathrm{I} . \mathrm{II}-\mathrm{I} .54, P=0.002)\end{array}$ & \\
\hline 3.2 & $\begin{array}{l}\text { ACM, coronary } \\
\text { events, } \\
\text { cerebrovascular } \\
\text { events }\end{array}$ & $\begin{array}{l}\text { Not } \\
\text { provided }\end{array}$ & $\begin{array}{l}24 \mathrm{~m} / \mathrm{s}, 14 \\
\mathrm{~m} / \mathrm{s}, 14 \mathrm{~m} / \mathrm{s}\end{array}$ & $\begin{array}{l}I .84(95 \% \mathrm{Cl} I .13- \\
2.88, P=0.016), I .69 \\
(95 \% \mathrm{Cl} I .06-2.84 \\
P=0.025), 1.63 \\
(95 \% \mathrm{Cl} I .0 \mathrm{I}-2.76 \\
P=0.046)\end{array}$ & Not provided & $\begin{array}{l}\text { For the CCVEs, } \\
\text { adjusted by modified } \\
\text { FRS }\end{array}$ \\
\hline
\end{tabular}

(Continued) 
Table 2 (Continued)

\begin{tabular}{|c|c|c|c|c|c|c|c|c|}
\hline Article & Index & Result & $\begin{array}{l}\text { Criteria of } \\
\text { ASO/PAD } \\
\text { exclusion }\end{array}$ & Device & Population & $\begin{array}{l}\text { Number } \\
\text { of } \\
\text { patients }\end{array}$ & $\begin{array}{l}\text { Age } \\
\text { (mean; } \\
\text { years) }\end{array}$ & $\begin{array}{l}\text { Male } \\
\text { gender } \\
(\%)\end{array}$ \\
\hline Kuroiwa et al ${ }^{49}$ & baPWV & Successful & $\begin{array}{l}\text { History of ASO, } \\
\mathrm{ABI}<0.9\end{array}$ & VP & $\begin{array}{l}\text { Community- } \\
\text { dwelling } \\
\text { elderly }\end{array}$ & 450 & 77 & 67.3 \\
\hline Sheng et $\mathrm{al}^{52}$ & baPWV & Successful & $A B \mid<0.9$ & VP & $\begin{array}{l}\text { Community- } \\
\text { dwelling } \\
\text { elderly }\end{array}$ & 3,876 & 68 & 44.2 \\
\hline Katakami et $\mathrm{al}^{53}$ & baPWV & Successful & PAD & VP & Diabetes & 1,040 & 59 & 65.0 \\
\hline Takashima et $\mathrm{al}^{58}$ & baPWV & Successful & $\mathrm{ABI}<0.9$ & VP & $\begin{array}{l}\text { General } \\
\text { population }\end{array}$ & 4,164 & 59 & 37.2 \\
\hline Ishisone et $\mathrm{al}^{60}$ & baPWV & Successful & $\mathrm{ABI} \leq 0.9$ & VP & $\begin{array}{l}\text { General } \\
\text { population }\end{array}$ & 973 & 59 & 46.9 \\
\hline Kawai et al ${ }^{61}$ & baPWV & Successful & $A B \mid<0.9$ & $\begin{array}{l}\text { FCP-473I } \\
\text { (Fukuda } \\
\text { Denshi) } \\
\end{array}$ & Hypertension & 440 & 61 & 56.1 \\
\hline Munakata et al ${ }^{63}$ & baPWV & Successful & PAD & VP & Hypertension & 662 & 60 & 45.4 \\
\hline Inoue et $\mathrm{al}^{65}$ & baPWV & Successful & PAD & VP & Hemodialysis & 197 & 66 & 61.9 \\
\hline Nakamura et al $^{67}$ & baPWV & Successful & $\mathrm{ABI}<0.9$ & VP & IHD & 564 & 64 & 80.9 \\
\hline Miyano et al ${ }^{69}$ & baPWV & Successful & $A B \mid<0.9$ & VP & $\begin{array}{l}\text { Community- } \\
\text { dwelling } \\
\text { elderly }\end{array}$ & 530 & 76 & 39.1 \\
\hline Meguro et $\mathrm{al}^{70}$ & baPWV & Successful & PAD & VP & $\mathrm{HF}$ & 72 & 68 & 56.9 \\
\hline Matsuoka et $\mathrm{al}^{71}$ & baPWV & Successful & $\mathrm{ABI} \leq 0.9$ & VP & $\begin{array}{l}\text { Community- } \\
\text { dwelling } \\
\text { elderly }\end{array}$ & 298 & 80 & 40.3 \\
\hline Park et $\mathrm{al}^{74}$ & baPWV & Failed & $A B \mid<0.9$ & VP & IHD & 203 & 57 & 52.7 \\
\hline Morimoto et $\mathrm{al}^{80}$ & baPWV & Failed & $A B \mid<0.9$ & VP & Hemodialysis & 176 & 61 & 56.3 \\
\hline Lee et al ${ }^{82}$ & baPWV & Successful & $A B \mid<0.9$ & VP & IHD & 350 & 66 & 53.4 \\
\hline Chen et $\mathrm{al}^{83}$ & baPWV & Failed & $A B \mid<0.9$ & VP & CKD & 227 & 65 & 43.0 \\
\hline Li et al $^{86}$ & baPWV & Failed & $\mathrm{ABI} \leq 0.9$ & VP & Outpatients & 238 & 69 & 42.9 \\
\hline Ueki et al ${ }^{97}$ & baPWV & Successful & $\mathrm{ABI} \leq 0.9, \mathrm{ABI}>\mathrm{I} .4$ & VP & $\begin{array}{l}\text { Outpatients, } \\
\text { CVDs }\end{array}$ & 2,554 & 66 & 70.2 \\
\hline
\end{tabular}




\begin{tabular}{|c|c|c|c|c|c|c|}
\hline $\begin{array}{l}\text { Follow- } \\
\text { up } \\
\text { (years) }\end{array}$ & $\begin{array}{l}\text { Primary end } \\
\text { point }\end{array}$ & $\begin{array}{l}\text { Number } \\
\text { of } \\
\text { events }\end{array}$ & Cutoff & HR (adjusted) & $\begin{array}{l}\text { HR as a } \\
\text { continuous } \\
\text { variable (adjusted) }\end{array}$ & Comments \\
\hline 3 & $\mathrm{ACM}$ & 28 & $18.61 \mathrm{~m} / \mathrm{s}$ & $\begin{array}{l}\text { OR } 3.22(95 \% \mathrm{Cl} \\
\text { I. } 26-8.22, P=0.014)\end{array}$ & $\begin{array}{l}\text { Per I m/s, OR I.I0 } \\
(95 \% \mathrm{Cl} I .00-1.21 \text {, } \\
P=0.047)\end{array}$ & $\begin{array}{l}\text { A multivariate logistic } \\
\text { regression analysis was } \\
\text { performed }\end{array}$ \\
\hline 5.9 & $\begin{array}{l}\text { ACM, death } \\
\text { of non- } \\
\text { cerebrovascular- } \\
\text { cardiovascular } \\
\text { cause }\end{array}$ & 316,168 & $23.3 \mathrm{~m} / \mathrm{s}$ & $\begin{array}{l}1.56(95 \% \mathrm{Cl} I .16- \\
2.08, P=0.003), 1.60 \\
(95 \% \mathrm{Cl} \text { I.I8-2.75, } \\
P=0.006)\end{array}$ & Failed & $\begin{array}{l}\text { Cox regression: the } \\
\text { top decile vs whole } \\
\text { population }\end{array}$ \\
\hline $\begin{array}{l}7.5 \\
\text { (median) }\end{array}$ & $\begin{array}{l}\text { CCVE + PAD } \\
(\mathrm{ABI}<0.9)\end{array}$ & 113 & $15.5 \mathrm{~m} / \mathrm{s}$ & Not provided & $\begin{array}{l}\text { Per I SD, I.33 } \\
(95 \% \mathrm{Cl} 1.09-1.62 \text {, } \\
P=0.004) \text { the SD is } \\
\text { not provided }\end{array}$ & \\
\hline $\begin{array}{l}6.5 \\
\text { (median) }\end{array}$ & CCVE & 40 & $18 \mathrm{~m} / \mathrm{s}$ & $\begin{array}{l}2.70(95 \% \mathrm{Cl} \\
1.18-6.19) / \mathrm{ss}<14 \\
\mathrm{~m} / \mathrm{s}, 6.94(95 \% \mathrm{Cl} \\
1.43-33.73)\end{array}$ & Failed & \\
\hline 7.8 & CCVE & 37 & $\begin{array}{l}\text { The top } \\
\text { decile }\end{array}$ & $\begin{array}{l}2.58(95 \% \mathrm{Cl} \\
\mathrm{I} .24-5.37, P=0.012)\end{array}$ & $\begin{array}{l}\text { Per I SD, I.47 } \\
(95 \% \mathrm{Cl} \text { I.09-I.98, } \\
P=0.01 \mathrm{I})\end{array}$ & \\
\hline 6.3 & CCVE & 62 & $17.5 \mathrm{~m} / \mathrm{s}$ & $\begin{array}{l}2.048(95 \% \mathrm{Cl} \\
\mathrm{I} .176-3.616, \\
P=0.0113)\end{array}$ & Not provided & \\
\hline 3 & CCVE & 24 & $17.5 \mathrm{~m} / \mathrm{s}$ & $\begin{array}{l}2.97(95 \% \mathrm{Cl} \\
\mathrm{I} .006-9.380)\end{array}$ & Not provided & $\begin{array}{l}\text { Nontreated } \\
\text { hypertension at the } \\
\text { recruitment }\end{array}$ \\
\hline 5.8 & CCVE & 89 & $\begin{array}{l}\text { Not } \\
\text { provided }\end{array}$ & Not provided & $\begin{array}{l}\text { Per I cm/s, I.046 } \\
(95 \% \mathrm{Cl} I .006-1.086, \\
P=0.0220)\end{array}$ & $\begin{array}{l}\text { In reality, the unit } \\
\text { is considered to be } \\
\text { meter per second }\end{array}$ \\
\hline $\begin{array}{l}2.1 \\
\text { (median) }\end{array}$ & CCVE & 122 & $\begin{array}{l}17.3 \mathrm{~m} / \mathrm{s} \text { for } \\
\text { the diabetes } \\
\text { patients only }\end{array}$ & $\begin{array}{l}\mathrm{I} .97(95 \% \mathrm{Cl} \\
\mathrm{I} .0 \mathrm{I}-3.84, P=0.046)\end{array}$ & Not provided & \\
\hline 3 & ACM, CCVM & 30,11 & $\begin{array}{l}19.63 \mathrm{~m} / \mathrm{s} \\
19.63 \mathrm{~m} / \mathrm{s}\end{array}$ & $\begin{array}{l}5.3(95 \% \mathrm{Cl} 2.2- \\
\mathrm{I} 2.7), 18.7(95 \% \mathrm{Cl} \\
2.2-157.6)\end{array}$ & $\begin{array}{l}\text { Per I m/s, I.09 (95\% } \\
\mathrm{Cl} \mathrm{I.00-I.I8),} \mathrm{I.I2} \\
(95 \% \mathrm{Cl} I .0 \mathrm{I}-\mathrm{I} .25)\end{array}$ & \\
\hline 1.2 & $\begin{array}{l}\text { Readmission of } \\
\text { HF exacerbation }\end{array}$ & 7 & $17.5 \mathrm{~m} / \mathrm{s}$ & $\begin{array}{l}5.101(95 \% \mathrm{Cl} \\
\mathrm{I}, 034-25.166, \\
P=0.045)\end{array}$ & Not provided & \\
\hline 3.4 & CCVM & 9 & $25 \mathrm{~m} / \mathrm{s}$ & Not provided & $\begin{array}{l}\text { Per } 2 \mathrm{~m} / \mathrm{s}, \mathrm{I} .302 \\
(95 \% \mathrm{Cl} \mathrm{I} . \mathrm{I} \mathrm{I} 0-\mathrm{I} .525, \\
P=0.00 \mathrm{II}) / \mathrm{per} 5 \mathrm{~m} / \mathrm{s}, \\
\mathrm{I} .933(95 \% \mathrm{Cl} \mathrm{I} .300- \\
2.874, P=0.00 \mathrm{II})\end{array}$ & \\
\hline 4.2 & CCVE & 36 & $\begin{array}{l}\text { Not } \\
\text { provided }\end{array}$ & Not provided & Failed & \\
\hline 3.6 & ACM, CCVM & 17,9 & $18 \mathrm{~m} / \mathrm{s}$ & Not provided & Failed & \\
\hline $\begin{array}{l}.2 \\
\text { (median) }\end{array}$ & CCVE & 21 & $17.9 \mathrm{~m} / \mathrm{s}$ & $\begin{array}{l}2.03(95 \% \mathrm{Cl} \\
\mathrm{I} .08-6.38, P=0.007)\end{array}$ & Not provided & \\
\hline 1.8 & CCVE & 28 & $\begin{array}{l}\text { Not } \\
\text { provided }\end{array}$ & Not provided & Failed & \\
\hline $\begin{array}{l}.7 \\
\text { (median) }\end{array}$ & $\mathrm{ACM}$ & 15 & $16 \mathrm{~m} / \mathrm{s}$ & Failed & Not provided & \\
\hline 5 & MACE & 133 & $16.44 \mathrm{~m} / \mathrm{s}$ & Not provided & $\begin{array}{l}\text { Per I m/s, I.I7 } \\
(95 \% \mathrm{Cl} I .04-\mathrm{I} .32, \\
P=0.0 \mathrm{II})\end{array}$ & $\begin{array}{l}\text { The cutoff of } 16.44 \\
\mathrm{~m} / \mathrm{s} \text { is significant } \\
\text { only in patients aged } \\
30-59 \text { years }\end{array}$ \\
\hline
\end{tabular}

(Continued) 
Table 2 (Continued)

\begin{tabular}{|c|c|c|c|c|c|c|c|c|}
\hline Article & Index & Result & $\begin{array}{l}\text { Criteria of } \\
\text { ASO/PAD } \\
\text { exclusion }\end{array}$ & Device & Population & $\begin{array}{l}\text { Number } \\
\text { of } \\
\text { patients }\end{array}$ & $\begin{array}{l}\text { Age } \\
\text { (mean; } \\
\text { years) }\end{array}$ & $\begin{array}{l}\text { Male } \\
\text { gender } \\
(\%)\end{array}$ \\
\hline Aisu et a ${ }^{98}$ & baPWV & Successful & $A B \mid<0.9$ & VP & Outpatients & 456 & $\begin{array}{l}71 \\
\text { (median) }\end{array}$ & 67.8 \\
\hline Lu et al ${ }^{103}$ & baPWV & Successful & $A B \mid<0.9$ & VP & $\begin{array}{l}\text { General } \\
\text { population }\end{array}$ & $4,25 I$ & 52 & 45.9 \\
\hline Tokitsu et al ${ }^{104}$ & baPWV & Successful & $A B I \leq 0.9$ & VP & $\begin{array}{l}\text { HF with } \\
\text { preserved } \\
\text { ejection } \\
\text { function }\end{array}$ & 426 & 71 & 55.3 \\
\hline Hwang et al ${ }^{105}$ & baPWV & Successful & $A B I<0.9$ & VP & $\begin{array}{l}\text { Suspected } \\
\text { IHD }\end{array}$ & 523 & 58 & 60.6 \\
\hline Sato et al ${ }^{109}$ & CAVI & Successful & $A B I<0.9$ & VS & Outpatients & 1,003 & 63 & 51.2 \\
\hline Kubota et $\mathrm{al}^{1 / 3}$ & CAVI & Successful & $A B \mid<0.9$ & VS & Outpatients & 400 & 69 & 63.0 \\
\hline
\end{tabular}

Abbreviations: ABI, ankle-brachial index; ACM, all-cause mortality; ACS, acute coronary syndrome; ASO, arteriosclerosis obliterans; baPWV, brachia-ankle pulse wave velocity; CAVI, cardio-ankle vascular index; CCVE, cerebrovascular-cardiovascular events; CCVM, cerebrovascular-cardiovascular mortality; CKD, chronic kidney disease; CVD, cardiovascular disease; FRS, Framingham risk score; HF, heart failure; IHD, ischemic heart disease; LE, lower extremity; MACE, major adverse cardiac events; PAD, peripheral arterial disease; VP, Vascular Profiler; VS, VaSera.

the progression of arterial stiffness and thus prognosis. ${ }^{118}$ However, the result of this study is plausible because this study explored the key factors for the success of prognostic prediction of baPWV and CAVI, not investigating the factors affecting arterial stiffness. This study also confirmed that more than half of the articles did not clarify the exclusion criteria of LE-ASO/PAD or did not exclude patients with LE-ASO/PAD when using baPWV and CAVI in the prognostic studies.

\section{baPWV}

\section{Main findings}

The success rate of baPWV articles (65.7\%) tended to be higher than that of CAVI articles $(40.0 \%)(P=0.083)$. This difference may be partly caused by the number of the study population. The success rate of baPWV articles $(75.9 \%)$ was similar to that of CAVI articles $(57.1 \%)$ in the studies clarifying the exclusion criteria of LE-ASO/ PAD. Among the 29 baPWV articles that had the patient exclusion criteria of LE-ASO/PAD, seven articles failed to prove prognostic significance of baPWV. Among the seven articles, two excluded those patients only with symptomatic PAD. ${ }^{27,45}$

The former study consisted of patients with multiple risk factors for cardiovascular disease (CVD), and the mean ABI in this cohort was $1.01 \pm 0.17$ (SD). Therefore, patients with an $\mathrm{ABI}$ of $\leq 0.9$ existed at high probability. In this study, baPWV, but not CAVI, showed statistical significance in the Kaplan-Meier analysis. Nevertheless, its significance was lost after multivariate adjustment by the Cox model including ABI as a covariate, and the cutoff ABI of 1.04 was selected as an independent predictor. ${ }^{27}$ Moreover, the mean baPWV and CAVI values of both sides were used in the analysis. This condition meant that the decreased baPWV or CAVI 


\begin{tabular}{|c|c|c|c|c|c|c|}
\hline $\begin{array}{l}\text { Follow- } \\
\text { up } \\
\text { (years) }\end{array}$ & $\begin{array}{l}\text { Primary end } \\
\text { point }\end{array}$ & $\begin{array}{l}\text { Number } \\
\text { of } \\
\text { events }\end{array}$ & Cutoff & HR (adjusted) & $\begin{array}{l}\text { HR as a } \\
\text { continuous } \\
\text { variable (adjusted) }\end{array}$ & Comments \\
\hline $\begin{array}{l}4.9 \\
\text { (median) }\end{array}$ & $\mathrm{HF}$ & 30 & $\begin{array}{l}\text { Not } \\
\text { provided }\end{array}$ & Not provided & $\begin{array}{l}\text { Per I m/s, I.2I } \\
(95 \% \mathrm{Cl} \text { I.I I-I.33, } \\
P<0.0 \mathrm{I}), \text { per } \\
\Delta \text { baPWV I0\%, I.5I } \\
(95 \% \mathrm{Cl} \text { I.23-I.86, } \\
P<0.0 \mathrm{I})\end{array}$ & \\
\hline $\begin{array}{l}4.4 \\
\text { (median) }\end{array}$ & CCVE & 74 & $\begin{array}{l}16.7 \mathrm{~m} / \mathrm{s} \\
\text { (Youden's } \\
\text { index) }\end{array}$ & $\begin{array}{l}\text { Unadjusted, II.2 } \\
(95 \% \mathrm{Cl} 6.59-19.1 \text {, } \\
P<0.000 \mathrm{I})\end{array}$ & $\begin{array}{l}\text { Per } 3.23 \mathrm{~m} / \mathrm{s}(\mathrm{I} \\
\mathrm{SD}), \mathrm{I} .50(95 \% \mathrm{Cl} \\
\mathrm{I} .26-\mathrm{I} .78, P \leq 0.000 \mathrm{I})\end{array}$ & $\begin{array}{l}\text { baPWV is adjusted by } \\
\text { heart rate such as } 75 \\
\text { beats per minute }\end{array}$ \\
\hline 2.8 & CCVE & 91 & $\begin{array}{l}<13 \mathrm{~m} / \mathrm{s} \text { (the } \\
\text { first quintile), } \\
19 \mathrm{~m} / \mathrm{s} \\
\leq<22 \mathrm{~m} / \mathrm{s} \\
\text { (the fourth } \\
\text { quintile), } 22 \\
\mathrm{~m} / \mathrm{s}<\text { (the } \\
\text { top quintile) }\end{array}$ & $\begin{array}{l}2.88(95 \% \mathrm{Cl} I .12- \\
7.38, P=0.03), 2.20 \\
(95 \% \mathrm{Cl} I .14-4.25, \\
P=0.02), 2.56(95 \% \\
C I I .28-5.14, \\
P=0.01)\end{array}$ & Not provided & \\
\hline 3.7 & CCVE & 66 & $16.19 \mathrm{~m} / \mathrm{s}$ & $\begin{array}{l}4.717(95 \% \mathrm{Cl} \\
2.675-8.319 \\
P<0.001)\end{array}$ & $\begin{array}{l}\text { Per I m/s, I.I29 } \\
(95 \% \mathrm{Cl} \text { I.074-I.187, } \\
P<0.00 \mathrm{I})\end{array}$ & \\
\hline 6.7 & $\begin{array}{l}\text { IHD + coronary } \\
\text { artery events } \\
\text { confirmed } \\
\text { by coronary } \\
\text { angiography }\end{array}$ & 90 & $\begin{array}{l}\text { I0.09 (the } \\
\text { top quartile) }\end{array}$ & Failed & $\begin{array}{l}\text { Per CAVI }=I, I .126 \\
(95 \% \mathrm{Cl} I .006-1.259 \\
P=0.039)\end{array}$ & \\
\hline 2.3 & CCVE & 49 & $\begin{array}{l}\geq 10 \text { (the top } \\
\text { tertile) }\end{array}$ & $\begin{array}{l}2.25(95 \% \mathrm{Cl} \\
1.02-4.95, P=0.04)\end{array}$ & Not provided & \\
\hline
\end{tabular}

on the side with asymptomatic PAD would lower the mean parameter. However, in reality, a patient was considered to have a high-risk prognosis. ${ }^{5,22,117,119}$ Therefore, the risk of a patient with asymptomatic PAD who was considered at equivalently high risk as a patient with symptomatic PAD was considerably underestimated by the falsely lowered baPWV or CAVI. ${ }^{27}$

In the study by Lau et $a 1,{ }^{45}$ the study cohort included patients with diabetes vintage of $15.2 \pm 7.5$ years. Thus, the high probability of a falsely overestimated ABI due to arterial calcification in the lower limbs was considered. ${ }^{5}$ The mean ABI of both sides, $1.1 \pm 0.1$, was used in the analysis, and the high probability of asymptomatic LE-ASO/PAD was considered. Furthermore, the mean baPWV of both sides was also used; as such, a similar phenomenon observed in the study by Kusunose et al would be most likely. ${ }^{27}$ As a result, no prognostic significance of baPWV was proven in this study. ${ }^{45}$ Thus, at least when utilizing baPWV and CAVI as prognostic predictors that include the lower-limb arteries in the measuring path, these findings imply that the exclusion of symptomatic PAD is insufficient. Therefore, redefining of these two articles and the reanalysis were performed, and this change presented the statistically higher success rate in the presence of exclusion criteria of LE-ASO/PAD than that in the absence of the criteria among the baPWV articles. At the same time, baPWV and exclusion of LE-ASO/PAD showed a statistical tendency in the multivariate logistic model (Table 5).

However, within the articles that clarified the exclusion criteria using ABI $(\leq 0.9)$ or an expression of LE-ASO/PAD exclusion, the other five studies did not prove an independent prognostic significance of baPWV. The cohorts of these studies were as follows: two patients with IHD, ${ }^{26,74}$ of the patients receiving hemodialysis, ${ }^{80}$ one of the patients 
Table 3 Details of the articles in which the prognostic predictability of baPWV and CAVI was analyzed in the same cohort

\begin{tabular}{|c|c|c|c|c|c|c|c|c|}
\hline Articles & Index & Result & $\begin{array}{l}\text { Exclusion } \\
\text { of ASO/ } \\
\text { PAD in } \\
\text { the LE }\end{array}$ & $\begin{array}{l}\text { Criteria of } \\
\text { ASO/PAD } \\
\text { exclusion }\end{array}$ & $\begin{array}{l}\text { Other } \\
\text { exclusion } \\
\text { criteria }\end{array}$ & $\begin{array}{l}\text { Usage of } \\
\text { baPWV- } \\
\text { CAVI }\end{array}$ & Device & Population \\
\hline Kato et $\mathrm{al}^{24}$ & $\begin{array}{l}\text { baPWV, } \\
\text { CAVI }\end{array}$ & $\begin{array}{l}\text { baPWV } \\
\text { Kaplan- } \\
\text { Meier, } \\
\text { CAVI } \\
\text { failed }\end{array}$ & No & & & Average & vS & Hemodialysis \\
\hline Kato et $\mathrm{a}^{25}$ & $\begin{array}{l}\text { baPWV, } \\
\text { CAVI }\end{array}$ & $\begin{array}{l}\text { baPWV } \\
\text { successful, } \\
\text { CAVI } \\
\text { failed }\end{array}$ & Yes & $\mathrm{ABI}<0.9$ & $\begin{array}{l}\text { Over } 76 \\
\text { years old }\end{array}$ & Higher & VS & Hemodialysis \\
\hline Otsuka et $\mathrm{al}^{26}$ & $\begin{array}{l}\text { baPWV, } \\
\text { CAVI }\end{array}$ & $\begin{array}{l}\text { baPWV } \\
\text { failed, } \\
\text { CAVI } \\
\text { successful }\end{array}$ & Yes & PAD & $\begin{array}{l}\mathrm{AF} \text {, other } \\
\text { various } \\
\text { criteria }\end{array}$ & & $\begin{array}{l}\text { baPWV } \\
\text { not } \\
\text { described, } \\
\text { CAVI VS }\end{array}$ & $\begin{array}{l}\text { IHD with } \\
\text { impaired } \\
\text { CAVI }\end{array}$ \\
\hline Kusunose et $\mathrm{al}^{27}$ & $\begin{array}{l}\text { baPWV, } \\
\text { CAVI }\end{array}$ & $\begin{array}{l}\text { baPWV } \\
\text { Kaplan- } \\
\text { Meier, } \\
\text { CAVI } \\
\text { failed }\end{array}$ & Yes & $\begin{array}{l}\text { Symptomatic } \\
\text { PAD }\end{array}$ & $\begin{array}{l}\text { End-stage } \\
\text { neoplasm, } \\
\text { etc. }\end{array}$ & Average & $\begin{array}{l}\text { baPWV } \\
\text { VP, CAVI } \\
\text { VS }\end{array}$ & $\begin{array}{l}\text { Outpatients } \\
\text { with two or } \\
\text { more risk } \\
\text { factors }\end{array}$ \\
\hline Gohbara et $\mathrm{al}^{28}$ & $\begin{array}{l}\text { baPWV, } \\
\text { CAVI }\end{array}$ & $\begin{array}{l}\text { baPWV } \\
\text { not } \\
\text { described, } \\
\text { CAVI } \\
\text { successful }\end{array}$ & Yes & PAD & $\begin{array}{l}\text { AF, aortic } \\
\text { diseases, } \\
\text { etc. }\end{array}$ & Average & $\begin{array}{l}\text { baPWV } \\
\text { VP, CAVI } \\
\text { VS }\end{array}$ & IHD, ACS \\
\hline
\end{tabular}

Abbreviations: $A B I$, ankle-brachial index; ACM, all-cause mortality; ACS, acute coronary syndrome; AF, atrial fibrillation; ASO, arteriosclerosis obliterans; baPWV, brachial-ankle pulse wave velocity; CAVI, cardio-ankle vascular index; CCVE, cerebrovascular-cardiovascular event; CCVM, cerebrovascular-cardiovascular mortality; IHD, ischemic heart disease; MACE, major adverse cardiac event; PAD, peripheral arterial disease; VP, Vascular Profiler; VS, VaSera.

A Comparison of the raw data

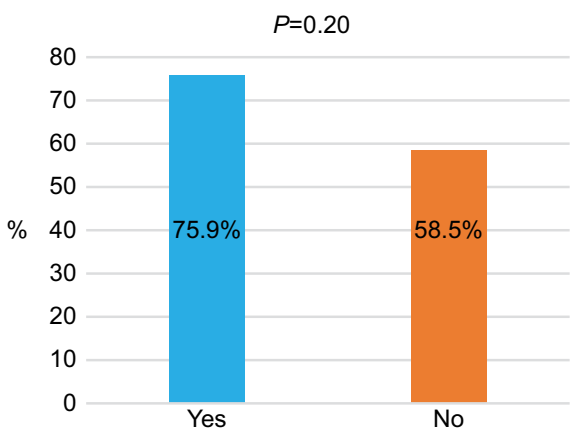

B Comparison after exchanging the two studies

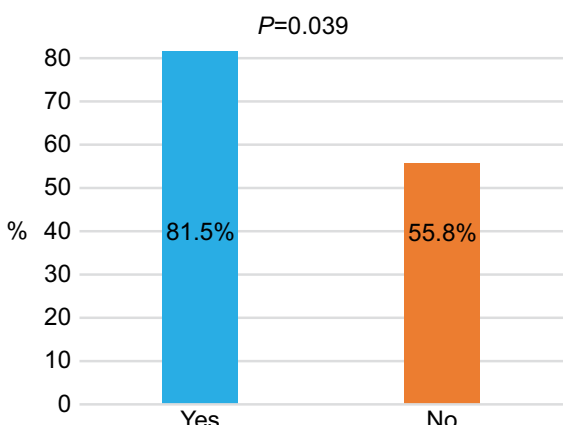

Figure 3 The success rate of baPWV articles according to the presence of exclusion criteria of LE-ASO/PAD. (A) Comparison of the raw data. (B) Comparison after exchanging the two studies.

Abbreviations: baPWV, brachial-ankle pulse wave velocity; LE-ASO/PAD, lower extremity-arteriosclerosis obliterans/peripheral arterial disease. 


\begin{tabular}{|c|c|c|c|c|c|c|c|c|c|}
\hline $\begin{array}{l}\text { Number } \\
\text { of } \\
\text { patients }\end{array}$ & $\begin{array}{l}\text { Age } \\
\text { (mean; } \\
\text { years) }\end{array}$ & $\begin{array}{l}\text { Male } \\
\text { gender } \\
(\%)\end{array}$ & $\begin{array}{l}\text { Follow- } \\
\text { up } \\
\text { (years) }\end{array}$ & $\begin{array}{l}\text { Primary } \\
\text { end } \\
\text { point }\end{array}$ & $\begin{array}{l}\text { Number } \\
\text { of } \\
\text { events }\end{array}$ & Cutoff & $\begin{array}{l}\text { HR } \\
\text { (adjusted) }\end{array}$ & $\begin{array}{l}\text { HR as a } \\
\text { continuous } \\
\text { variable } \\
\text { (adjusted) }\end{array}$ & Comments \\
\hline 194 & 64 & 65.5 & 3.3 & $\begin{array}{l}\text { ACM, } \\
\text { nonfatal } \\
\text { CCVE }\end{array}$ & 39,15 & $\begin{array}{l}\text { baPWV I } 7.5 \\
\mathrm{~m} / \mathrm{s} \text { (the top } \\
\text { tertile), CAVI } \\
\mathrm{I} 0.7 \text { (the top } \\
\text { tertile) }\end{array}$ & Failed & $\begin{array}{l}\text { Not } \\
\text { provided }\end{array}$ & $\begin{array}{l}\text { ABI succeeded } \\
\text { significantly }\end{array}$ \\
\hline 135 & 60 & 67.4 & 5.3 & CCVM & 22 & $\begin{array}{l}\text { baPWV I6.6 } \\
\mathrm{m} / \mathrm{s} \text { (the top } \\
\text { tertile), CAVI } \\
9.9 \text { (the top } \\
\text { tertile) }\end{array}$ & $\begin{array}{l}16.9 \\
(95 \% \mathrm{Cl} \\
\mathrm{I} .1-251.8 \\
P=0.04)\end{array}$ & $\begin{array}{l}\text { Not } \\
\text { provided }\end{array}$ & \\
\hline 211 & 65 & 55.9 & 2.9 & CCVE & 28 & $\begin{array}{l}\text { baPWV at } \\
\text { the second } \\
\text { occasion, } \\
\text { persistently } \\
\text { impaired, } \\
\text { CAVI in } 6 \\
\text { months }\end{array}$ & $\begin{array}{l}\text { baPWV } \\
\text { failed, } \\
\text { persistently } \\
\text { impaired } \\
\text { CAVI } 3.3 \\
(95 \% \text { Cl } \\
1.47-8.59 \text {, } \\
P<0.01)\end{array}$ & $\begin{array}{l}\text { Not } \\
\text { provided }\end{array}$ & $\begin{array}{l}\text { CAVI at the } \\
\text { first occasion } \\
\text { did not } \\
\text { succeed }\end{array}$ \\
\hline 114 & 69 & 78.1 & 4.3 & MACE & 35 & $\begin{array}{l}\text { baPWV } \\
=17.03 \mathrm{~m} / \mathrm{s}, \\
\text { CAVI }=9.2\end{array}$ & $\begin{array}{l}\text { Not } \\
\text { provided }\end{array}$ & Failed & $\begin{array}{l}A B I=I .0 I \pm 0.17 \\
(S D)\end{array}$ \\
\hline 288 & 65 & 82.3 & $\begin{array}{l}\text { I.3 } \\
\text { (median) }\end{array}$ & CCVE & 19 & $\begin{array}{l}\text { baPWV, not } \\
\text { provided, } \\
\text { CAVI } 8.325\end{array}$ & $\begin{array}{l}\text { baPWV not } \\
\text { provided, } \\
\text { CAVI I8 } \\
(95 \% \mathrm{Cl} \\
2.369- \\
136.8 \\
P=0.005)\end{array}$ & $\begin{array}{l}\text { Not } \\
\text { provided }\end{array}$ & $\begin{array}{l}\text { In the } \\
\text { secondary end } \\
\text { point (nonfatal } \\
\text { ischemic } \\
\text { stroke), CAVI } \\
\text { succeeded, } \\
\text { baPWV failed }\end{array}$ \\
\hline
\end{tabular}

Table 4 Logistic regression analysis exploring the independent determinants of the success of prognostic prediction

\begin{tabular}{|l|l|l|l|l|}
\hline & \multicolumn{2}{l|}{ Univariate } & \multicolumn{2}{l|}{ Multivariate } \\
\hline Covariates & OR (95\% Cl) & $P$-value & OR (95\% CI) & \\
\hline baPWV (yes =I) & $2.87(0.92-9.03)$ & $0.07 I$ & & \\
\hline Exclusion of ASO/PAD (yes =I) & $2.30(0.92-5.77)$ & 0.076 & & \\
\hline Dialysis population (yes =I) & $0.19(0.06-0.60)$ & 0.005 & $0.28(0.08-0.94)$ & \\
\hline Age (years) & $0.99(0.93-1.07)$ & 0.87 & & \\
\hline Male gender (\%) & $1.00(0.97-1.03)$ & 0.97 & & \\
\hline Follow-up period (years) & $1.00(0.78-1.27)$ & 0.97 & II.20 (2.45-5I.70) & \\
\hline Log (number of patients) & $14.00(3.13-62.80)$ & 0.0006 & 0.002 \\
\hline
\end{tabular}

Notes: baPWV $=\mathrm{I}$ or $\mathrm{CAVI}=0$ is used as a binary variate. The "success or failure" in the studies simultaneously comparing baPWV and CAVI was defined for each index. Thus, the total number of the included studies is 85 .

Abbreviations: ASO, arteriosclerosis obliterans; baPWV, brachial-ankle pulse wave velocity; CAVI, cardio-ankle vascular index; PAD, peripheral arterial disease.

with chronic kidney disease stage $3-5,{ }^{83}$ and one of an outpatient population with a $78 \%$ incidence of diabetes. ${ }^{86}$ Thus, these studies were conducted in population that were still very likely to include patients with LE-ASO/
PAD (false-negative LE-ASO/PAD), even if the exclusion criterion was set at $A B I \leq 0.9$. Therefore, the reason for failure in these five studies is considerably similar to that in the two studies. ${ }^{27,45}$ The reason why ABI and/or (false- 
Table 5 Logistic regression analysis exploring the independent determinants of the success of prognostic prediction after exchanging the studies excluding patients with only symptomatic PAD

\begin{tabular}{|c|c|c|c|c|}
\hline \multirow[b]{2}{*}{ Covariates } & \multicolumn{2}{|l|}{ Univariate } & \multicolumn{2}{|l|}{ Multivariate } \\
\hline & OR (95\% Cl) & $P$-value & OR (95\% Cl) & $P$-value \\
\hline baPWV (yes = I) & $2.87(0.92-9.03)$ & 0.071 & $3.36(0.86-13.20)$ & 0.083 \\
\hline Exclusion of ASO/PAD (yes $=1$ ) & $3.71(1.37-10.10)$ & 0.01 & $3.08(0.96-9.93)$ & 0.060 \\
\hline Dialysis population (yes =I) & $0.19(0.06-0.60)$ & 0.005 & $0.27(0.07-0.96)$ & 0.043 \\
\hline Age (years) & $0.99(0.93-1.07)$ & 0.87 & & \\
\hline Male gender (\%) & $1.00(0.97-1.03)$ & 0.97 & & \\
\hline Follow-up period (years) & $1.00(0.78-1.27)$ & 0.97 & & \\
\hline Log (number of patients) & $14.00(3.13-62.80)$ & 0.0006 & $9.04(1.90-43.00)$ & 0.006 \\
\hline
\end{tabular}

Notes: baPWV $=\mathrm{I}$ or CAVI $=0$ is used as a binary variate. The "success or failure" in the studies simultaneously comparing baPWV and CAVI was defined for each index. Thus, the total number of the included studies is 85 .

Abbreviations: ASO, arteriosclerosis obliterans; baPWV, brachia-ankle pulse wave velocity; CAVI, cardio-ankle vascular index; PAD, peripheral arterial disease.

negative) LE-ASO/PAD is a stronger indicator of prognosis is given in the following section.

\section{Prognostic significance of $A B I$ is much stronger than that of baPWV without sufficient exclusion of LE- ASO/PAD}

In the hemodialysis cohort that did not exclude patients with LE-ASO/PAD, it was already demonstrated that a high baPWV (the top quartile of baPWV $\geq 23.6 \mathrm{~m} / \mathrm{s}$ ) lost prognostic significance after multivariate adjustment including ABI as one of the covariates using the Cox model, even though a high baPWV showed significance in the Kaplan-Meier analysis. ${ }^{46}$ This is because ABI, which assesses LE-ASO/PAD as a more severe disease, is a considerably stronger prognostic indicator than baPWV. In the same study, baPWV showed independent prognostic significance after the exclusion of patients with an ABI of $<0.9$. Nevertheless, patients with a borderline ABI of $0.90-0.99$ and patients with a high ABI of $\geq 1.3$ also showed independent prognostic significance. Furthermore, patients with a low normal ABI of 1.00-1.09 showed a statistical tendency or a close value as a prognostic indicator $(P=0.113$ for $\mathrm{ACM}, P=0.086$ for $\mathrm{CCVM}$ after adjustment). ${ }^{46}$

In another study of hemodialysis patients evaluating the prognostic significance of $\mathrm{ABI}$ but not using baPWV, ${ }^{120}$ patients with an abnormal $\mathrm{ABI}$ of $<0.9$ and those with a borderline ABI of 0.90-0.99 showed the worst prognosis. However, in the present study, those with a high ABI of $\geq 1.3$ and even those with a low normal ABI of 1.00-1.09 showed significantly worse prognosis than those with a reference $A B I$ of 1.10-1.29 (in those with a low normal ABI of 1.00-1.09, an HR of $1.92 \%$ and $95 \%$ CI of $1.02-3.59$ for ACM and an HR of $2.82 \%$ and $95 \%$ CI of $1.22-6.54$ for CCVM after multivariate adjustment using the Cox proportional hazards model). In the report published in 2003 , Ono et al ${ }^{120}$ mentioned that those with an ABI of 0.9-1.1 as well as those with an abnormal ABI should be carefully monitored in the hemodialysis population.

Moreover, in a study of a hemodialysis cohort that evaluated the prognostic predictability of $\mathrm{ABI}$ in ACM, the best cutoff $\mathrm{ABI}$ of 1.1 was demonstrated by a receiver operating characteristic (ROC) curve analysis (area under the ROC curve to predict mortality, 0.79 ; sensitivity, 0.90 ; specificity, 0.62). ${ }^{121}$ This significance was maintained after multivariate analysis using the Cox model. In the studies that evaluated the diagnostic ability of ABI compared to imaging modalities or clinical symptoms in patients on hemodialysis, an ABI threshold of $1.01-1.10$ was mainly reported. ${ }^{122-124}$ Ohtake et $\mathrm{al}^{123}$ suggested raising the ABI cutoff to 1.1 in patients on hemodialysis. In a cohort of patients with diabetes, significant HRs and $P$-values in prognostic predictability (ACM and CCVM) were reportedly similar between those with an abnormal ABI of $\leq 0.9$ and those with a borderline ABI of 0.91-0.99 (both HRs of about 2.0, significant) compared to those with normal ABI of $1.00-1.4 .{ }^{125}$ Moreover, in a study of a cohort with multiple cardiovascular risk factors and a history of CVDs, those with a borderline ABI of 0.91-0.99 showed significantly higher HRs in ACM (HR 2.27, $P=0.005$ ) and CCVM (HR 3.47, $P=0.003$ ) than those with a normal ABI of 1.00-1.4. ${ }^{126}$

Among the studies of patients with IHD, one report showed the independent prognostic predictability of a borderline ABI of 0.91-0.99, ${ }^{127}$ whereas another study demonstrated the best cutoff $\mathrm{ABI}$ of 1.057 as an independent prognostic predictor. ${ }^{128}$ In contrast, in the Hisayama study involving a general population, those with abnormal $\mathrm{ABI}$ of $\leq 0.9$ clearly 
showed independent prognostic predictability in CCVE (HR 2.40, $P=0.02$ ). However, those with a borderline ABI of 0.91-0.99 did not show any difference compared to those with a normal ABI of 1.0-1.4, and the result was virtually the same even in the Kaplan-Meier analysis. ${ }^{129}$ All the ABI values in the study mentioned earlier were measured using VP. The information described earlier indicated that there are frequent cases in which those with LE-ASO/PAD still exist after excluding those with an ABI of $\leq 0.9$ and that a borderline or low normal $\mathrm{ABI}$ has stronger prognostic significance than baPWV depending on the cohort or at least has confounding power to weaken the prognostic significance of baPWV in the multivariate analysis. Moreover, it is also plausible that the existence of the patients with false-negative LE-ASO/PAD weakens the prognostic significance of baPWV even if $\mathrm{ABI}$ is not included in the multivariate model, because the prognostic risk of such a patient cannot be appropriately assessed by baPWV even if the higher baPWV is used. ${ }^{117}$ Simply, in other words, the existence of the false-negative LE-ASO/ PAD weakens the prognostic significance of baPWV (and also CAVI) anyway regardless of the ABI included in the multivariate model or not.

\section{Appropriate settings when evaluating baPWV and CAVI}

Therefore, to set the exclusion criteria of LE-ASO/PAD to appropriately assess baPWV as a prognostic indicator, the cutoff $\mathrm{ABI}$ of $\leq 0.9$ is sometimes insufficient, or it would be necessary to increase the ABI value in such a case. This is one of the limitations when using baPWV (and also CAVI). As such, among the seven studies that failed to show the prognostic significance of baPWV, ${ }^{26,27,45,74,80,83,86}$ if the exclusion criteria of LE-ASO/PAD were defined to include the upstroke time $(\mathrm{UT})^{130}$ and/or percent mean arterial pressure (\%MAP), ${ }^{131}$ the baPWV success rate would be higher. This might be the same for CAVI. In contrast, several studies showed the independent prognostic significance of baPWV and CAVI without the clarified exclusion criteria of LE-ASO/ PAD or without LE-ASO/PAD exclusion. For this reason, some studies might have excluded LE-ASO/PAD using the cutoff $\mathrm{ABI}$ of $\leq 0.9$, but it might not be just precisely described in the articles (it is very likely in three articles of reference number of 56, 68, and 111 for some reasons). These studies might also be performed in the cohort with a low frequency of LE-ASO/PAD even if LE-ASO/PAD was not excluded. It is also plausible that the independent prognostic predictability of baPWV or CAVI was proven incidentally in the relationship of the covariates included in the multivariate model.
In reality, two baPWV ${ }^{56,68}$ and one $\mathrm{CAVI}^{111}$ studies, which were defined as not clarifying exclusion criteria of LE-ASO/ PAD, were considered most likely to exclude those with LE$\mathrm{ASO} / \mathrm{PAD}$ and/or $\mathrm{ABI}$ of $\leq 0.9$. It is almost certain according to the data of $\mathrm{ABI}(\mathrm{ABI}=1.13 \pm 0.00$ [standard error], the number of patients was 338), ${ }^{56}$ the context of the patient exclusion criteria and the end point, ${ }^{111}$ and the information of other studies of their institutions. ${ }^{58,68}$ Therefore, reanalysis was performed. Among the baPWV articles, the success rate of the articles in the presence of these criteria $(82.8 \%$ [24/29]) was significantly higher than that of the articles in the absence of these criteria $(53.7 \%[22 / 41] ; P=0.020)$. In the multivariate logistic regression analysis (Table $\mathrm{S} 1$ ), exclusion of LE-ASO/PAD emerged as an independent predictor of the successful prognostic prediction $(P=0.022)$. The $P$-value of baPWV improved (from 0.083 to 0.059 ).

\section{CAVI}

\section{Main findings}

The success rate tended to be lower in studies of CAVI than in those of baPWV (40\% vs $65.7 \%, P=0.083$ ). Moreover, all four studies of a hemodialysis cohort failed to show prognostic significance. ${ }^{24,25,108,116}$ In three of the four studies, LE-ASO/PAD was not excluded. ${ }^{24,106,114}$ The prognostic significance of CAVI was also definitely weakened by uncertainty or absence of the exclusion criteria of LE-ASO/ PAD. The relatively small number of the participants in the hemodialysis studies might also affect the outcome. However, among the studies clarifying the exclusion criteria of LE-ASO/PAD, the success rate was $57.1 \%(4 / 7)$, which was lower than that for studies of baPWV (75.9\%), although this was not statistically significant. Moreover, four of the six studies that showed the independent prognostic significance of CAVI implied that the statistical power was not very strong from the aspect of $P$-values despite the studied cohorts not being very small $(P=0.039$ for 1,003 patients; $P=0.029$ for 300 patients; $P=0.049$ or $<0.05$ for 626 patients; $P=0.04$ for 400 patients $).{ }^{109,111-113}$ Furthermore, the largest study of CAVI involving 2,106 Caucasian participants with metabolic syndromes failed. In this study, there were no clarified criteria of LE-ASO/PAD. Nevertheless, the subjects were middle aged (54 \pm 6 years), and those with the previous history of CVDs were excluded. Thus, the prevalence of LE-ASO/PAD was considered low in this cohort. In the present study, CAVI showed statistical significance in the Kaplan-Meier analysis and the univariate Cox model. However, in the multivariate analysis, this significance was lost, and age and gender were selected as 
the independent prognostic predictors. ${ }^{110}$ These phenomena are similar to that of the hemodialysis study by Kato et al. ${ }^{25}$ In Kato's study, the statistical tendency of CAVI was lost after the adjustment of age, gender, and diabetes in the multivariate Cox proportional hazards model.

Therefore, considering the whole information described earlier, the prognostic power of CAVI may be weaker than that of baPWV. The possible reasons are described in the following section.

\section{Factors possibly affecting prognostic predictability of CAVI}

CAVI is a product of PWV adjusted by blood pressure, and its concept is derived from the stiffness parameter $\beta .{ }^{14}$ In adopting the concept of the stiffness parameter $\beta$ on heart-ankle PWV (haPWV), the equation is as follows: $\beta=\mathrm{CAVI}=2 \rho^{\circ}$ $\mathrm{PP}^{-1 \cdot} \mathrm{Ln}(\mathrm{SBP} / \mathrm{DBP})^{\cdot}$ haPWV, ${ }^{2}$ where $\rho$ is the blood density, $\mathrm{PP}$ is the pulse pressure, and $\mathrm{Ln}$ is a natural logarithm. ${ }^{14} \mathrm{As}$ a result of this method, CAVI is considered less dependent on blood pressure than baPWV or independent from blood pressure. ${ }^{14,132}$ However, the appropriateness of using only brachial blood pressure as the representative of the haPWV measuring path, as well as the independence of blood pressure, has been controversial. ${ }^{132-141} \mathrm{~A}$ few authors in these studies clearly denied the blood pressure independence of CAVI. ${ }^{135,138,139}$ To calculate haPWV, the pulse transit times between the brachial-ankle and the heart-brachial are required. The equation of haPWV is as follows: haPWV $=\mathrm{Lha} / \mathrm{Tha}=\mathrm{Lha} /$ $(\mathrm{Tba}+\mathrm{Thb})$, where Lha is the length between the heart and the ankle, Tha is the pulse transit time between the heart and the ankle, Tba is the pulse transit time between the brachium and the ankle, and Thb is the pulse transit time between the heart and the brachium. ${ }^{14}$ However, measuring Tha or Thb is virtually impossible. Therefore, it is substituted with the time interval between the onset of the second heart sound and that of the dicrotic notch (DN) on the brachial pulse wave form (Thb $\left.\fallingdotseq \mathrm{T}^{\prime} \mathrm{hb}=\mathrm{TII}-\mathrm{DN}\right)$. Nevertheless, one study reported that this method using volume plethysmography could induce a $50 \%$ reduction in CAVI. ${ }^{142}$ The timings of the second sound and the DN themselves could be falsely determined depending on the patient's condition, especially in cases of valvular heart diseases. Furthermore, the blood density $\rho$ is considered constant in the VS device, but this is not always constant in vivo. Kato et $\mathrm{al}^{25}$ pointed out the change in blood density of patients on hemodialysis. ${ }^{143}$ Moreover, we must recognize the risk of using the brachial blood pressure of the upper extremity (UE)-ASO in those on hemodialysis. Patients on hemodialysis reportedly have falsely elevated ABI to a certain extent because of UE-ASO on the contralateral side of the hemodialysis access, and its frequency was reportedly about $10 \%$ in the Japanese hemodialysis patients. ${ }^{17,144} \mathrm{In}$ all four hemodialysis cohort studies that failed to show the prognostic significance of CAVI, no difference in CAVI was found between those with and without the primary end point (before adjustment). This might be caused by the absence of exclusion criteria of LE-ASO/PAD in three of the studies. Nevertheless, a decreased brachial blood pressure due to UE-ASO and error in the pulse transit time ( $\mathrm{T}^{\prime} \mathrm{hb}$ and Tba) might have partly affected the results. We must also recognize that a false measurement of $\mathrm{ABI}$ and baPWV in the cases of UE-ASO in patients on hemodialysis would affect the prognostic predictability of baPWV. ${ }^{117}$

With respect to blood pressure and $\mathrm{ABI}$ measurement, we must recognize that there are a few minor differences between VP and VS. VP synchronizes the timing of SBP determination to make it simultaneous. In contrast, a blood pressure measurement using VS is basically performed through the sequential method (from the right side to the left side). Thus, for determining SBPs in the arms and ankles, the difference in measurement time is considered more likely to occur with VS than with VP. In the statement document of the American Heart Association published in 2012 regarding the measurement and interpretation of $\mathrm{ABI}$, two studies reported that the left $\mathrm{ABI}$ measured using the Doppler method was significantly lower $(0.03)$ than the right ABI. ${ }^{5}$ In a meta-analysis of the risks of inter-arm blood pressure differences, a significantly increased relative risk of inter-arm difference (ie, difference in $\mathrm{SBP} \geq 10 \mathrm{mmHg}$ ) was demonstrated in the sequential method compared to the simultaneous method. ${ }^{145}$ The difference in the risk of the inter-arm blood pressure difference mentioned earlier may affect the individual ABI and the prevalence of LEASO/PAD (it could also change the excluded patients) and may affect the brachial blood pressure to be used in the CAVI equation. Furthermore, as a fundamental issue, we may have to ensure that the blood pressure measured by the oscillometric method is used in the CAVI equation of VS. Blood pressures measured by the oscillometric method are reportedly lower than those measured by the invasive method (internal arterial pressure). ${ }^{146-148}$ This implies that, even if it is theoretically correct to use the brachial blood pressure as a representative value of the systemic arteries in the CAVI equation, because it is an oscillometric method anyway, a discrepancy inevitably exists regardless of the device used. It should also be recognized that the invasive method is not always perfect. 


\section{Difference between CAVI and baPWV}

Various factors that would affect the prognostic significance of CAVI were discussed earlier. The correlation between baPWV and haPWV, which is a parameter in the CAVI equation, is reportedly very high in healthy male individuals of the general population ( $r=0.92$ for baPWV and haPWV in the right side, $\mathrm{n}=135$; mean, 59 years old).$^{149}$ In contrast, the blood pressure dependence of CAVI is reportedly weaker than that of baPWV..$^{14,132}$ Moreover, several studies have shown superior associations with other atherosclerotic parameters of CAVI to that of baPWV. ${ }^{150,151}$ However, we may have to recognize that the characteristics of PWV, which is considered to natively possess prognostic significance, ${ }^{6-8,18-21}$ might be affected by various factors in the measurement and the equation of CAVI as pointed out earlier. Furthermore, we suppose that CAVI is superior to baPWV as an index of arterial wall stiffness. Nevertheless, we may also have to recognize that the superiority for quantifying arterial wall stiffness itself is a different issue from superiority as a prognostic predictor. We may also have to recognize a study that showed the superiority of baPWV to CAVI in terms of reproducibility in the Caucasian population, although the statistical difference was not described. ${ }^{152}$

\section{Perspective}

\section{Necessity of more prognostic studies of CAVI}

Three meta-analyses of the prognostic significance of baPWV have already been published, ${ }^{18-21}$ and the cutoff baPWV of 18 $\mathrm{m} / \mathrm{s}$ is largely consistent. ${ }^{3,21,22}$ No published meta-analysis has examined the prognostic significance of CAVI probably due to the shortage of reports. Thus, further studies are required. The large-scale CAVI-J study that aims to validate the prognostic significance of CAVI is currently in progress, and its results are pending. ${ }^{153} \mathrm{~A}$ few large longitudinal studies in Western countries are also in progress. According to the MARK study in Spain, CAVI was significantly and positively associated with an index of physical functional quality of life (standardized physical component: the higher, the better), ABI was also significantly and positively associated (both after multivariate adjustment), and baPWV was not correlated. ${ }^{154}$ Moreover, baPWV and CAVI showed similar correlations with carotid atherosclerosis indices. ${ }^{155}$ This result is similar to that of the Japanese hemodialysis study. ${ }^{143}$ In the comparison of cfPWV and CAVI according to the Advanced Approach to Arterial Stiffness study of 18 European countries, age-gender adjustment of cfPWV but not CAVI was higher in the patients with metabolic syndrome than those without. ${ }^{141,156} \mathrm{~A}$ similar result was also reported in Japan. In the present study, baPWV but not CAVI was significantly higher in the patients with metabolic syndromes than those without among the middle-aged health checkup population. ${ }^{157}$ It will be interesting to note whether the same difference in study results as that obtained in the Japanese cohorts is demonstrated. ${ }^{136,154,156}$

\section{Necessity of rigorous patient exclusion criteria}

When using baPWV and CAVI, especially in a study cohort consisting of patients with severe conditions, the patient exclusion criteria should be more rigorous; at least, LE-ASO/ PAD definition of $A B I \leq 0.9$ should be used. Arrhythmia and aortic valve disease should be used as well. ${ }^{117}$ In addition, UE-ASO should be considered in patients receiving hemodialysis. ${ }^{117,144}$ Especially, regarding the exclusion criteria of LE-ASO/PAD in the study of prognostic significance, as the first step, we can set the best ABI cutoff by ROC curve analysis in the prognostic prediction. The analysis of baPWV and CAVI can be permitted only in patients with an ABI that exceeds the best cutoff. When using a simple cutoff such as $\mathrm{ABI} \leq 0.9$ or $\mathrm{ABI} \leq 0.99$, the heart rate-adjusted $\mathrm{UT}^{130}$ and $\% \mathrm{MAP}^{86,131}$ should also be included in the criteria. ${ }^{158}$ Moreover, the use of a higher baPWV and CAVI on either side is favorable. ${ }^{22,119}$ Especially, when using the mean or designated side of those indices, the masked LE-ASO/PAD must be thoroughly excluded.

\section{Adopting the concept of stiffness parameter $\beta$ and other methods of blood pressure adjustment}

The concept of stiffness $\beta$ and $\mathrm{CAVI}^{14}$ can be applied to other devices that measure PWV and blood pressure if we neglect the "a" and "b" constants, which are used to convert the slope and the coordinate of the CAVI equation in VS. We can also use a general constant for the blood density $\rho$. In fact, a few studies have adopted this idea using VP. ${ }^{159}$ However, this is not exactly the same as the CAVI measured by VS. Nevertheless, a baPWV-derived CAVI, the brachial-ankle vascular index, is comparable to the baPWV, and this conversion is quite easy to make. Therefore, a reanalysis comparing both indices in the previously published studies would be interesting. If necessary, haPWV can also be measured by changing the VP settings. ${ }^{149}$ Regarding CAVI, Spronck et al ${ }^{138}$ suggested a novel method of adjusting blood pressure. Steppan et al ${ }^{135}$ also suggested "arterial stiffness index," which is the product of PWV divided by PP (PWV/PP), as an effective method to adjust the influence of blood pressure on PWV. One study indicated a strong linear relationship between baPWV and the sum of four-limb PP. ${ }^{160}$ Applying those concepts to these devices would also be easy. 


\section{Necessity of prospective large-scale study}

Finally, this study discussed the independent prognostic predictability of baPWV and CAVI after adjustment by the multivariate Cox proportional hazards model. The more important ability is the additive predictive value on conventional risk factors (reclassification improvement in risk stratification), which was demonstrated in a few studies of baPWV and CAVI. ${ }^{47,111}$ This was also demonstrated in the latest meta-analysis of baPWV using individual participant data. ${ }^{20}$ Further studies are expected to confirm the superior index including this factor. Furthermore, no study to date directly compared prognostic predictability of baPWV and cfPWV in a same study population. Therefore, a prospective large-scale study is warranted to simultaneously investigate baPWV, CAVI, cfPWV, and other arterial stiffness indices in the prognostic significance.

\section{Limitations}

There are several limitations to the interpretation of the results. First, because the number of articles was insufficient, the multivariate logistic analysis showed only the statistical tendency for the significance of baPWV after redefining the studies. Moreover, the analysis also demonstrated the most powerful effect of the number of the participants in each study. This implies that the success of the prognostic prediction strongly relies upon the quality of the study itself. However, it should be recognized that the statistical tendency for baPWV and exclusion criteria of LE-ASO/PAD emerged in the number of currently available articles. The results also imply that the reproducibility of baPWV as a prognostic predictor is superior to that of CAVI in the various clinical conditions. Moreover, the fact that baPWV already showed results similar to those for cfPWV in the meta-analyses would be consistent with the results of this study as a whole. Furthermore, we should recognize that only $40 \%$ of the studies proved the prognostic significance of CAVI. Second, publication bias was not considered in this study. As such, denying the existence of unpublished studies that could affect the statistical results is difficult. Nevertheless, the ratio of articles that clarified the exclusion criteria of LE-ASO/PAD was similar in the baPWV and CAVI studies. The success rates of the baPWV and CAVI studies declined in the absence of clarified exclusion criteria of LE-ASO/PAD. Moreover, the ratio of the articles studying the prognostic significance of these parameters did not differ significantly (baPWV 70/1,800; CAVI 15/550). Therefore, the prognostic studies of baPWV and CAVI were published without strong bias. Third, this study did not consider other criteria of the patient exclu- sion such as arrhythmia and aortic valve diseases. However, the description and the definition of the patient exclusion criteria are diverse among each study and sometimes uncertain. Thus, it was impossible to quantitatively include these factors in the statistical analysis. Nevertheless, the major limitation when using baPWV and CAVI is the presence of LE-ASO/PAD. Therefore, this factor was representatively included in the analysis. Fourth, this study did not consider the difference of covariates entered into the Cox multivariate model in each study. It is possible that the success or failure of prognostic prediction of baPWV and CAVI are induced by missing covariates or inappropriate adjustment. However, most articles researched in this study were peer reviewed. Therefore, the incidence of the inappropriate multivariate analysis would be low. Fifth, this study considered seven parameters potentially included in the multivariate logistic analysis. There might be other important factors that should have been included. Nevertheless, the number of the successful studies in the prognostic prediction was 52 . Therefore, it was difficult to increase the number of parameters anyway. Finally, PubMed was the only database used in this study. However, PubMed is a widely used database worldwide, so most of the English-written articles related to the theme of this study are included.

\section{Conclusion}

This study demonstrated that the number of study participants and dialysis population were the independent determinants of the successful prognostic prediction in the baPWV and CAVI articles. This study also showed that baPWV tended to be superior to CAVI in the prognostic prediction. Moreover, the exclusion criteria of LE-ASO/PAD also affected the prognostic predictive success of both indices. Therefore, for the appropriate use of these indices, thorough LE-ASO/PAD exclusion is essential. In addition, a large-scale prospective study to simultaneously research the prognostic significance of these indices is warranted.

\section{Acknowledgment}

The author appreciates the researchers and participants of the preceding studies.

\section{Disclosure}

Dai Ato is a former employee of Fukuda Colin (formerly Omron Colin, Nippon Colin) Co., Ltd. Fukuda Colin is a distributor of VP (form PWV/ABI, BP-203RPE series). Dai Ato wrote this article as an academic activity based on the guaranteed right of freedom in academia for the Japanese 
(Article 23) and on the supreme law provided in Article 98 of the Constitution of Japan. Dai Ato reports no other conflicts of interest in this work.

\section{References}

1. Bramwell JC, Hill AV. Velocity of transmission of the pulse-wave: and elasticity of arteries. Lancet. 1922;199(5149):891-892.

2. Yao ST, Hobbs JT, Irvine WT. Ankle pressure measurement in arterial disease of the lower extremities. Br J Surg. 1968;55(11):859-860.

3. Vlachopoulos C, Xaplanteris P, Aboyans V, et al. The role of vascular biomarkers for primary and secondary prevention. A position paper from the European Society of Cardiology Working Group on peripheral circulation: Endorsed by the Association for Research into Arterial Structure and Physiology (ARTERY) Society. Atherosclerosis. 2015;241(2):507-532.

4. Townsend RR, Wilkinson IB, Schiffrin EL, et al. Recommendations for Improving and Standardizing Vascular Research on Arterial Stiffness: A Scientific Statement From the American Heart Association. Hypertension. 2015;66(3):698-722.

5. Aboyans V, Criqui MH, Abraham P, et al. Measurement and interpretation of the ankle-brachial index: a scientific statement from the American Heart Association. Circulation. 2012;126(24):2890-2909.

6. Vlachopoulos C, Aznaouridis K, Stefanadis C. Prediction of cardiovascular events and all-cause mortality with arterial stiffness: a systematic review and meta-analysis. J Am Coll Cardiol. 2010;55:1318-1327.

7. Ben-Shlomo Y, Spears M, Boustred C, et al. Aortic pulse wave velocity improves cardiovascular event prediction: an individual participant meta-analysis of prospective observational data from 17,635 subjects. J Am Coll Cardiol. 2014;63(7):636-646.

8. Zhong Q, Hu MJ, Cui YJ, et al. Carotid-Femoral Pulse Wave Velocity in the Prediction of Cardiovascular Events and Mortality: An Updated Systematic Review and Meta-Analysis. Angiology. 2018;69(7):617-629.

9. Suzuki E, Kashiwagi A, Nishio Y, et al. Increased arterial wall stiffness limits flow volume in the lower extremities in type 2 diabetic patients. Diabetes Care. 2001;24(12):2107-2114.

10. Yamashina A, Tomiyama H, Takeda K, et al. Validity, reproducibility, and clinical significance of noninvasive brachial-ankle pulse wave velocity measurement. Hypertens Res. 2002;25(3):359-364.

11. Yambe T, Kovalev YA, Milyagina IA, et al. A Japanese-Russian collaborative study on aging and atherosclerosis. Biomed Pharmacother. 2004;58(Suppl 1):S91-S94.

12. Orlova I, Kuz'mina AE, Barinova IV, Iarovaia EB, Ageev FT. Assessment of major artery stiffness: new perspectives of noninvasive diagnosis of coronary atherosclerosis. Ter Arkh. 2009;81(4):8-13.

13. Yambe T, Yoshizawa M, Saijo Y, et al. Brachio-ankle pulse wave velocity and cardio-ankle vascular index (CAVI). Biomed Pharmacother. 2004;58(Suppl 1):S95-S98.

14. Shirai K, Utino J, Otsuka K, Takata M. A novel blood pressureindependent arterial wall stiffness parameter; cardio-ankle vascular index (CAVI). J Atheroscler Thromb. 2006;13(2):101-107.

15. Tomiyama H, Koji Y, Yambe M, et al. Brachial -- ankle pulse wave velocity is a simple and independent predictor of prognosis in patients with acute coronary syndrome. Circ J. 2005;69(7):815-822.

16. Yamamoto N, Yamanaka G, Ishikawa M, et al. Cardio-ankle vascular index as a predictor of cognitive impairment in community-dwelling elderly people: four-year follow-up. Dement Geriatr Cogn Disord. 2009;28(2):153-158.

17. Ato D, Takami T. Brachial-Ankle Pulse Wave Velocity, Mortality, and Cardiovascular Events. J Cardiovasc Disord. 2015;2(1):1009.

18. Vlachopoulos C, Aznaouridis K, Terentes-Printzios D, Ioakeimidis N, Stefanadis C. Prediction of cardiovascular events and all-cause mortality with brachial-ankle elasticity index: a systematic review and meta-analysis. Hypertension. 2012;60(2):556-562.
19. Tomiyama H, Matsumoto C, Shiina K, Yamashina A. Brachial-Ankle PWV: Current Status and Future Directions as a Useful Marker in the Management of Cardiovascular Disease and/or Cardiovascular Risk Factors. J Atheroscler Thromb. 2016;23(2):128-146.

20. Ohkuma T, Ninomiya T, Tomiyama H, et al. Brachial-Ankle Pulse Wave Velocity and the Risk Prediction of Cardiovascular Disease: An Individual Participant Data Meta-Analysis. Hypertension. 2017;69(6):1045-1052.

21. Ohkuma T, Tomiyama H, Ninomiya T, et al. Proposed Cutoff Value of Brachial-Ankle Pulse Wave Velocity for the Management of Hypertension. Circ J. 2017;81(10):1540-1542.

22. Yamashina A, Kario K, Kohara K. Guidelines for noninvasive vascular function test (JCS); 2013. Available from: http://www.j-circ.or.jp/ guideline/pdf/JCS2013_yamashina_h.pdf. Accessed October 5, 2018.

23. Shimamoto K, Ando K, Fujita T. Japanese Society of Hypertension Committee for Guidelines for the Management of Hypertension. The Japanese Society of Hypertension Committee for Guidelines for the Management of Hypertension. Hypertens Res. 2014;37(4):253-390.

24. Kato A, Takita T, Furuhashi M, Kumagai H, Hishida A. A small reduction in the ankle-brachial index is associated with increased mortality in patients on chronic hemodialysis. Nephron Clin Pract. 2010;114(1):c29-c37.

25. Kato A, Takita T, Furuhashi M, Maruyama Y, Miyajima H, Kumagai H. Brachial-ankle pulse wave velocity and the cardio-ankle vascular index as a predictor of cardiovascular outcomes in patients on regular hemodialysis. Ther Apher Dial. 2012;16(3):232-241.

26. Otsuka K, Fukuda S, Shimada K, et al. Serial assessment of arterial stiffness by cardio-ankle vascular index for prediction of future cardiovascular events in patients with coronary artery disease. Hypertens Res. 2014;37(11):1014-1020.

27. Kusunose K, Sato M, Yamada H, et al. Prognostic Implications of Non-Invasive Vascular Function Tests in High-Risk Atherosclerosis Patients. Circ J. 2016;80(4):1034-1040.

28. Gohbara M, Iwahashi N, Sano Y, et al. Clinical Impact of the CardioAnkle Vascular Index for Predicting Cardiovascular Events After Acute Coronary Syndrome. Circ J. 2016;80(6):1420-1426.

29. Kusunose K, Yamada H. Noninvasive Vascular Function Tests - Long Journey for Predicting Cardiovascular Events. Circ J. 2016;80(6):1321-1322.

30. Shimizu T, Yoshihisa A, Kanno Y, et al. Relationship of hyperuricemia with mortality in heart failure patients with preserved ejection fraction. Am J Physiol Heart Circ Physiol. 2015;309(7):H1123-H1129.

31. Lin CY, Leu JG, Fang YW, Tsai MH. Association of interleg difference of ankle brachial index with overall and cardiovascular mortality in chronic hemodialysis patients. Ren Fail. 2015;37(1):88-95.

32. Tokitsu T, Yamamoto E, Hirata Y, et al. Clinical significance of pulse pressure in patients with heart failure with preserved left ventricular ejection fraction. Eur J Heart Fail. 2016;18(11):1353-1361.

33. Suzuki H, Inoue T, Dogi M, Kikuta T, Takenaka T, Okada H. Role of Pulse Wave Velocity in Patients with Chronic Kidney Disease Stages 3-5 on Long-Term Follow-Up. Pulse. 2015;2(1-4):1-10.

34. Huang JC, Chen SC, Su HM, Chang JM, Hwang SJ, Chen HC. Performance of the Framingham risk score in patients receiving hemodialysis. Nephrology. 2013;18(7):510-515.

35. Ohtake T, Ishioka K, Honda K, et al. Impact of coronary artery calcification in hemodialysis patients: Risk factors and associations with prognosis. Hemodial Int. 2010;14(2):218-225.

36. Miura T, Minamisawa M, Ueki Y, et al. Impressive predictive value of ankle-brachial index for very long-term outcomes in patients with cardiovascular disease: IMPACT-ABI study. PLoS One. 2017;12(6):e0177609.

37. Minamisawa M, Miura T, Motoki H, et al. Geriatric Nutritional Risk Index Predicts Cardiovascular Events in Patients at Risk for Heart Failure. Circ J. 2018;82(6):1614-1622.

38. Iimori S, Mori Y, Akita W, et al. Effects of sevelamer hydrochloride on mortality, lipid abnormality and arterial stiffness in hemodialyzed patients: a propensity-matched observational study. Clin Exp Nephrol. 2012;16(6):930-937. 
39. Yoshihisa A, Suzuki S, Yamaki T, et al. Impact of adaptive servo-ventilation on cardiovascular function and prognosis in heart failure patients with preserved left ventricular ejection fraction and sleep-disordered breathing. Eur J Heart Fail. 2013;15(5): 543-550.

40. Sugimoto T, Yamamoto K, Takizawa K, et al. Assessment of pulse wave velocity as a marker of post-operative cardiovascular risk in off-pump coronary artery bypass grafting patients. Kyobu Geka. 2010;63(7):531-535.

41. Ichikawa K, Sakuragi S, Nishihara T, et al. Influence of arterial stiffness on cardiovascular outcome in patients without high blood pressure. Heart. 2018;104(4):318-323.

42. Kanda Y. Investigation of the freely available easy-to-use software 'EZR' for medical statistics. Bone Marrow Transplant. 2013;48(3):452-458.

43. Yoshida M, Mita T, Yamamoto R, et al. Combination of the Framingham risk score and carotid intima-media thickness improves the prediction of cardiovascular events in patients with type 2 diabetes. Diabetes Care. 2012;35(1):178-180.

44. Tokitsu T, Yamamoto E, Hirata Y, et al. Clinical significance of pulse pressure in patients with coronary artery disease. Int $J$ Cardiol. 2015;190:299-301.

45. Lau KK, Wong YK, Chan YH, et al. Prognostic implications of surrogate markers of atherosclerosis in low to intermediate risk patients with type 2 diabetes. Cardiovasc Diabetol. 2012;11(1):101.

46. Kitahara T, Ono K, Tsuchida A, et al. Impact of brachial-ankle pulse wave velocity and ankle-brachial blood pressure index on mortality in hemodialysis patients. Am J Kidney Dis. 2005;46(4): 688-696.

47. Ninomiya T, Kojima I, Doi Y, et al. Brachial-ankle pulse wave velocity predicts the development of cardiovascular disease in a general Japanese population: the Hisayama Study. J Hypertens. 2013;31(3):477-483

48. Maeda Y, Inoguchi T, Etoh E, et al. Brachial-ankle pulse wave velocity predicts all-cause mortality and cardiovascular events in patients with diabetes: the Kyushu Prevention Study of Atherosclerosis. Diabetes Care. 2014;37(8):2383-2390.

49. Kuroiwa Y, Miyano I, Nishinaga M, et al. Association between level of brachial-ankle pulse wave velocity and onset of activities of daily living impairment in community-dwelling older individuals. Geriatr Gerontol Int. 2015;15(7):840-847.

50. Seo HJ, Ki YJ, Han MA, Choi DH, Ryu SW. Brachial-ankle pulse wave velocity and mean platelet volume as predictive values after percutaneous coronary intervention for long-term clinical outcomes in Korea: A comparable and additive study. Platelets. 2015;26(7):665-671.

51. Chang LH, Lin HD, Kwok CF, et al. The combination of the ankle brachial index and brachial ankle pulse wave velocity exhibits a superior association with outcomes in diabetic patients. Intern Med. 2014;53(21):2425-2431.

52. Sheng CS, Li Y, Li LH, et al. Brachial-ankle pulse wave velocity as a predictor of mortality in elderly Chinese. Hypertension. 2014;64(5):1124-1130.

53. Katakami N, Osonoi T, Takahara M, et al. Clinical utility of brachial-ankle pulse wave velocity in the prediction of cardiovascular events in diabetic patients. Cardiovasc Diabetol. 2014;13(1): 128.

54. Ki YJ, Choi DH, Lee YM, Lim L, Song H, Koh YY. Predictive value of brachial-ankle pulse wave velocity for long-term clinical outcomes after percutaneous coronary intervention in a Korean cohort. Int $J$ Cardiol. 2014;175(3):554-559.

55. Kim J, Song TJ, Song D, et al. Brachial-ankle pulse wave velocity is a strong predictor for mortality in patients with acute stroke. Hypertension. 2014;64(2):240-246.

56. Kawai T, Ohishi M, Onishi M, et al. Prognostic impact of regional arterial stiffness in hypertensive patients. Heart Vessels. 2015;30(3):338-346.
57. Nagai K, Shibata S, Akishita M, et al. Efficacy of combined use of three non-invasive atherosclerosis tests to predict vascular events in the elderly; carotid intima-media thickness, flow-mediated dilation of brachial artery and pulse wave velocity. Atherosclerosis. 2013;231(2):365-370.

58. Takashima N, Turin TC, Matsui K, et al. The relationship of brachialankle pulse wave velocity to future cardiovascular disease events in the general Japanese population: the Takashima Study. J Hum Hypertens. 2014;28(5):323-327.

59. Yoon HE, Shin DI, Kim SJ, et al. Brachial-ankle pulse wave velocity predicts decline in renal function and cardiovascular events in early stages of chronic kidney disease. Int J Med Sci. 2013;10(11):1430-1436.

60. Ishisone T, Koeda Y, Tanaka F, Sato K, Nagano M, Nakamura M. Comparison of utility of arterial stiffness parameters for predicting cardiovascular events in the general population. Int Heart J. 2013;54(3): 160-165.

61. Kawai T, Ohishi M, Onishi M, et al. Cut-off value of brachial-ankle pulse wave velocity to predict cardiovascular disease in hypertensive patients: a cohort study. J Atheroscler Thromb. 2013;20(4): 391-400.

62. Han JY, Choi DH, Choi SW, et al. Predictive value of brachialankle pulse wave velocity for cardiovascular events. Am J Med Sci. 2013;346(2):92-97.

63. Munakata M, Konno S, Miura Y, Yoshinaga K; J-TOPP Study Group. Prognostic significance of the brachial-ankle pulse wave velocity in patients with essential hypertension: final results of the J-TOPP study. Hypertens Res. 2012;35(8):839-842.

64. Chen SC, Chang JM, Liu WC, et al. Brachial-ankle pulse wave velocity and rate of renal function decline and mortality in chronic kidney disease. Clin J Am Soc Nephrol. 2011;6(4):724-732.

65. Inoue T, Ogawa T, Ishida $\mathrm{H}$, Ando $\mathrm{Y}$, Nitta $\mathrm{K}$. Aortic arch calcification evaluated on chest $\mathrm{X}$-ray is a strong independent predictor of cardiovascular events in chronic hemodialysis patients. Heart Vessels. 2012;27(2):135-142.

66. Orlova I, Kuz'mina AE, Masenko VP, Iarovaia EB, Ageev FT. Effect of arterial stiffness on development of cardio-vascular complications in ischemic heart disease. Kardiologiia. 2009;49(12):11-17.

67. Nakamura M, Yamashita T, Yajima J, et al. Brachial-ankle pulse wave velocity as a risk stratification index for the short-term prognosis of type 2 diabetic patients with coronary artery disease. Hypertens Res. 2010;33(10):1018-1024.

68. Turin TC, Kita Y, Rumana N, et al. Brachial-ankle pulse wave velocity predicts all-cause mortality in the general population: findings from the Takashima study, Japan. Hypertens Res. 2010;33(9):922-925.

69. Miyano I, Nishinaga M, Takata J, et al. Association between brachialankle pulse wave velocity and 3-year mortality in community-dwelling older adults. Hypertens Res. 2010;33(7):678-682.

70. Meguro T, Nagatomo Y, Nagae A, et al. Elevated arterial stiffness evaluated by brachial-ankle pulse wave velocity is deleterious for the prognosis of patients with heart failure. Circ J. 2009;73(4): 673-680.

71. Matsuoka O, Otsuka K, Murakami S, et al. Arterial stiffness independently predicts cardiovascular events in an elderly community -- Longitudinal Investigation for the Longevity and Aging in Hokkaido County (LILAC) study. Biomed Pharmacother. 2005;59(Suppl 1):S40-S44.

72. Wang X, Dang A. Prognostic Value of Brachial-Ankle Pulse Wave Velocity in Patients With Takayasu Arteritis With Drug-Eluting Stent Implantation. Arthritis Care Res. 2015;67(8):1150-1157.

73. Chen SC, Lee WH, Hsu PC, et al. Association of Brachial-Ankle Pulse Wave Velocity With Cardiovascular Events in Atrial Fibrillation. Am J Hypertens. 2016;29(3):348-356.

74. Park KH, Han SJ, Kim HS, et al. Impact of Framingham risk score, flow-mediated dilation, pulse wave velocity, and biomarkers for cardiovascular events in stable angina. J Korean Med Sci. 2014;29(10):1391-1397. 
75. Kuwahara M, Hasumi S, Mandai S, et al. Rate of ankle-brachial index decline predicts cardiovascular mortality in hemodialysis patients. Ther Apher Dial. 2014;18(1):9-18.

76. Orlova IA, Nuraliev EY, Yarovaya EB, Ageev FT. Prognostic value of changes in arterial stiffness in men with coronary artery disease. Vasc Health Risk Manag. 2010;6:1015-1021.

77. Tanaka M, Ishii H, Aoyama T, et al. Ankle brachial pressure index but not brachial-ankle pulse wave velocity is a strong predictor of systemic atherosclerotic morbidity and mortality in patients on maintenance hemodialysis. Atherosclerosis. 2011;219(2):643-647.

78. Amemiya N, Ogawa T, Otsuka K, Ando Y, Nitta K. Comparison of serum albumin, serum C-reactive protein, and pulse wave velocity as predictors of the 4-year mortality of chronic hemodialysis patients. $J$ Atheroscler Thromb. 2011;18(12):1071-1079.

79. Chen SC, Chang JM, Tsai JC, et al. A new systolic parameter defined as the ratio of brachial pre-ejection period to brachial ejection time predicts overall and cardiovascular mortality in hemodialysis patients. Hypertens Res. 2010;33(5):492-498.

80. Morimoto S, Yurugi T, Aota Y, et al. Prognostic significance of anklebrachial index, brachial-ankle pulse wave velocity, flow-mediated dilation, and nitroglycerin-mediated dilation in end-stage renal disease. Am J Nephrol. 2009;30(1):55-63.

81. Nagata Y, Miura S, Suematsu Y, et al. Association between major adverse cardiovascular events and brachial-ankle pulse wave velocity and a difference in blood pressure between arms after percutaneous coronary intervention. Exp Clin Cardiol. 2014.

82. Lee HS, Kim HL, Kim H, et al. Incremental Prognostic Value of Brachial-Ankle Pulse Wave Velocity to Single-Photon Emission Computed Tomography in Patients with Suspected Coronary Artery Disease. J Atheroscler Thromb. 2015;22(10):1040-1050.

83. Chen SC, Chang JM, Tsai JC, et al. A systolic parameter defined as the ratio of brachial pre-ejection period to brachial ejection time predicts cardiovascular events in patients with chronic kidney disease. Circ J. 2010;74(10):2206-2210.

84. Wei SY, Huang JC, Chen SC, Chang JM, Chen HC. Unequal Arterial Stiffness With Overall and Cardiovascular Mortality in Patients Receiving Hemodialysis. Am J Med Sci. 2016;351 (2):187-193

85. Iino R, Yokoyama N, Konno K, Naito K, Isshiki T. Impact of combined assessment of coronary artery calcium score, carotid artery plaque score, and brachial-ankle pulse wave velocity for early coronary revascularization in patients with suspected coronary artery disease. Int Heart J. 2012;53(3):154-159.

86. Li YH, Lin SY, Sheu WH, Lee IT. Relationship between percentage of mean arterial pressure at the ankle and mortality in participants with normal ankle-brachial index: an observational study. BMJOpen. 2016;6(3):e010540

87. Sugamata W, Nakamura T, Uematsu M, et al. The combined assessment of flow-mediated dilation of the brachial artery and brachialankle pulse wave velocity improves the prediction of future coronary events in patients with chronic coronary artery disease. J Cardiol. 2014;64(3):179-184.

88. Song Y, Xu B, Xu R, et al. Independent and Joint Effect of BrachialAnkle Pulse Wave Velocity and Blood Pressure Control on Incident Stroke in Hypertensive AdultsNovelty and Significance. Hypertension. 2016;68(1):46-53.

89. Ikura K, Hanai K, Oka S, et al. Brachial-ankle pulse wave velocity, but not ankle-brachial index, predicts all-cause mortality in patients with diabetes after lower extremity amputation. J Diabetes Investig. 2017;8(2):250-253.

90. Kawai T, Ohishi M, Takeya Y, et al. Adiponectin single nucleotide polymorphism is a genetic risk factor for stroke through high pulse wave pressure: a cohort study. J Atheroscler Thromb. 2013;20(2):152-160.

91. Chen SC, Chang JM, Tsai YC, et al. Association of interleg BP difference with overall and cardiovascular mortality in hemodialysis. Clin J Am Soc Nephrol. 2012;7(10):1646-1653.
92. Mimura T, Takenaka T, Kanno Y, Moriwaki K, Okada H, Suzuki H. Vascular compliance is secured under angiotensin inhibition in non-diabetic chronic kidney diseases. J Hum Hypertens. 2008;22(1):38-47.

93. Ahn KT, Jeong JO, Jin SA, et al. Brachial-ankle PWV for predicting clinical outcomes in patients with acute stroke. Blood Press. 2017;26(4):204-210

94. Kim EJ, Choi MJ, Lee JH, et al. Extracellular Fluid/Intracellular Fluid Volume Ratio as a Novel Risk Indicator for All-Cause Mortality and Cardiovascular Disease in Hemodialysis Patients. PLoS One. 2017;12(1):e0170272.

95. Tabata N, Sueta D, Yamashita T, et al. Relationship between asymptomatic intra-cranial lesions and brachial-ankle pulse wave velocity in coronary artery disease patients without stroke. Hypertens Res. 2017;40(4):392-398.

96. Saji N, Murotani K, Shimizu H, et al. Increased pulse wave velocity in patients with acute lacunar infarction doubled the risk of future ischemic stroke. Hypertens Res. 2017;40(4):371-375.

97. Ueki Y, Miura T, Minamisawa M, et al. The usefulness of brachial-ankle pulse wave velocity in predicting long-term cardiovascular events in younger patients. Heart Vessels. 2017;32(6):660-667.

98. Aisu H, Saito M, Inaba S, et al. Association of worsening arterial stiffness with incident heart failure in asymptomatic patients with cardiovascular risk factors. Hypertens Res. 2017;40(2):173-180.

99. Kuo TH, Yang DC, Lin WH, et al. Compliance Index, a Marker of Peripheral Arterial Stiffness, may Predict Renal Function Decline in Patients with Chronic Kidney Disease. Int J Med Sci. 2015;12(7):530-537.

100. Kajimoto T, Sawamura MS, Hayashi RD, Oya T, Hirao RA, Kouhara $\mathrm{H}$. High efficient and cost-effective screening method for diabetic cardiovascular risk. Diabetol Metab Syndr. 2014;6(1):51.

101. Woo JS, Kim W, Jang HH, Kim JB, Kim WS, Kim KS. Effect of platelet reactivity, endothelial function, and inflammatory status on outcomes in patients with stable angina pectoris on clopidogrel therapy. Am J Cardiol. 2014;113(5):786-792.

102. Park HW, Kang MG, Kim K, et al. Prognostic value of brachial-ankle pulse wave velocity in patients with non-ST-elevation myocardial infarction. Coron Artery Dis. 2017;28(8):642-648.

103. Lu YC, Lyu P, Zhu HY, et al. Brachial-ankle pulse wave velocity compared with mean arterial pressure and pulse pressure in risk stratification in a Chinese population. J Hypertens. 2018;36(3):528-536.

104. Tokitsu T, Yamamoto E, Oike F, et al. Clinical significance of brachial-ankle pulse-wave velocity in patients with heart failure with preserved left ventricular ejection fraction. J Hypertens. 2018;36(3):560-568.

105. Hwang IC, Jin KN, Kim HL, et al. Additional prognostic value of brachial-ankle pulse wave velocity to coronary computed tomography angiography in patients with suspected coronary artery disease. Atherosclerosis. 2018;268:127-137.

106. Wang LL, Luo Q, Zhu BX, Zhou FF. Brachial-Ankle Pulse Wave Velocity Could Be a Predictor of Mortality in Patients on Peritoneal Dialysis. Perit Dial Int. 2018;38(3):215-219.

107. Taniguchi Y, Kitamura A, Shinozaki T, et al. Trajectories of arterial stiffness and all-cause mortality among community-dwelling older Japanese. Geriatr Gerontol Int. 2018;18(7):1108-1113.

108. Yamaguchi S, Gohda T, Gotoh H, et al. Factors associated with cardiovascular death and events in patients with end stage renal disease. Nihon Jinzo Gakkai Shi. 2013;55(2):159-166.

109. Sato Y, Nagayama D, Saiki A, et al. Cardio-Ankle Vascular Index is Independently Associated with Future Cardiovascular Events in Outpatients with Metabolic Disorders. J Atheroscler Thromb. 2016;23(5):596-605.

110. LauceviČius A, Ryliškytè L, Balsytè J, et al. Association of cardio-ankle vascular index with cardiovascular risk factors and cardiovascular events in metabolic syndrome patients. Medicina. 2015;51(3):152-158. 
111. Satoh-Asahara N, Kotani K, Yamakage H, et al. Cardio-ankle vascular index predicts for the incidence of cardiovascular events in obese patients: a multicenter prospective cohort study (Japan Obesity and Metabolic Syndrome Study: JOMS). Atherosclerosis. 2015;242(2):461-468.

112. Chung SL, Yang CC, Chen CC, Hsu YC, Lei MH. Coronary Artery Calcium Score Compared with Cardio-Ankle Vascular Index in the Prediction of Cardiovascular Events in Asymptomatic Patients with Type 2 Diabetes. J Atheroscler Thromb. 2015;22(12): $1255-1265$.

113. Kubota Y, Maebuchi D, Takei M, et al. Cardio-Ankle Vascular Index is a predictor of cardiovascular events. Artery Res. 2011;5(3):91-96.

114. Yoshihisa A, Takiguchi M, Shimizu T, et al. Cardiovascular function and prognosis of patients with heart failure coexistent with chronic obstructive pulmonary disease. J Cardiol. 2014;64(4):256-264.

115. Sawai A, Yasuda Y, Suzuki S, et al. Impact of non-invasive cardiovascular screening programs as a predictor of cardiovascular events among asymptomatic chronic kidney disease patients. Clin Exp Nephrol. 2016;20(3):416-424.

116. Harada M, Tsukada W, Tsukada O, Hashimoto K, Kamijo Y. The Optimal Cut-off Value of Ankle Brachial Index for Screening Cardiovascular Disease Risk in Hemodialysis Patients. Shinshu Med J,. 2016;64(3):135-146.

117. Ato D. Pitfalls in the ankle-brachial index and brachial-ankle pulse wave velocity. Vasc Health Risk Manag. 2018;14:41-62.

118. Ciccone MM, Bilianou E, Balbarini A, et al. Task force on: 'Early markers of atherosclerosis: influence of age and sex'. $J$ Cardiovasc Med. 2013;14(10):757-766.

119. Munakata M. Brachial-ankle pulse wave velocity in the measurement of arterial stiffness: recent evidence and clinical applications. Curr Hypertens Rev. 2014;10(1):49-57.

120. Ono K, Tsuchida A, Kawai H, et al. Ankle-brachial blood pressure index predicts all-cause and cardiovascular mortality in hemodialysis patients. J Am Soc Nephrol. 2003;14(6):1591-1598.

121. Otani Y, Otsubo S, Kimata N, et al. Effects of the ankle-brachial blood pressure index and skin perfusion pressure on mortality in hemodialysis patients. Intern Med. 2013;52(21):2417-2421.

122. Ogata H, Kumata-Maeta C, Shishido K, et al. Detection of peripheral artery disease by duplex ultrasonography among hemodialysis patients. Clin J Am Soc Nephrol. 2010;5(12):2199-2206.

123. Ohtake T, Oka M, Ikee R, et al. Impact of lower limbs' arterial calcification on the prevalence and severity of PAD in patients on hemodialysis. J Vasc Surg. 2011;53(3):676-683.

124. Tsuyuki K, Kohno K, Ebine K, et al. Usefulness of ExerciseAnkle Brachial Pressure Index Test and Its Diagnostic Criteria in Patients on Maintenance Hemodialysis. J Japanese College Angiol. 2015;55(2):21-25.

125. Natsuaki C, Inoguchi T, Maeda Y, et al. Association of borderline anklebrachial index with mortality and the incidence of peripheral artery disease in diabetic patients. Atherosclerosis. 2014;234(2):360-365.

126. Tanaka S, Kaneko H, Kano H, et al. The predictive value of the borderline ankle-brachial index for long-term clinical outcomes: An observational cohort study. Atherosclerosis. 2016;250:69-76.

127. Lee SH, Choi SH, Kim EK, et al. Borderline ankle-brachial index is associated with poor short-term clinical outcome after coronary artery intervention. Atherosclerosis. 2016;249:186-190.

128. Kim HL, Seo JB, Chung WY, Zo JH, Kim MA, Kim SH. Prognostic value of the ankle-brachial index in patients undergoing drug-eluting stent implantation. $J$ Atheroscler Thromb. 2015;22(1):27-37.

129. Kojima I, Ninomiya T, Hata J, et al. A low ankle brachial index is associated with an increased risk of cardiovascular disease: the Hisayama study. J Atheroscler Thromb. 2014;21(9):966-973.

130. Sheng CS, Li Y, Huang QF, Kang YY, Li FK, Wang JG. Pulse Waves in the Lower Extremities as a Diagnostic Tool of Peripheral Arterial Disease and Predictor of Mortality in Elderly Chinese. Hypertension. 2016;67(3):527-534.
131. Hashimoto T, Ichihashi S, Iwakoshi S, Kichikawa K. Combination of pulse volume recording (PVR) parameters and ankle-brachial index (ABI) improves diagnostic accuracy for peripheral arterial disease compared with ABI alone. Hypertens Res. 2016;39(6): 430-434.

132. Shirai K, Song M, Suzuki J, et al. Contradictory effects of $\beta 1$ - and $\alpha 1$ - adrenergic receptor blockers on cardio-ankle vascular stiffness index (CAVI)--CAVI independent of blood pressure. $J$ Atheroscler Thromb. 2011;18(1):49-55.

133. Tomiyama H, Yamashina A. Central blood pressure--a possible latent factor affecting CAVI. J Atheroscler Thromb. 2011;18(8):720; author reply $721-722$.

134. Shirai K, Takahashi M. Author's Reply to: Central Blood Pressure: A Possible Latent Factor Affecting CAVI. J Atheroscler Thromb. 2011;18(8):721-722.

135. Steppan J, Sikka G, Hori D, et al. Seeking a blood pressure-independent measure of vascular properties. Hypertens Res. 2016;39(1): 27-38.

136. Maliha G, Townsend RR. A study of the VaSera arterial stiffness device in US patients. J Clin Hypertens. 2017;19(7):661-668.

137. Lim J, Pearman ME, Park W, Alkatan M, Machin DR, Tanaka H. Impact of blood pressure perturbations on arterial stiffness. Am J Physiol Regul Integr Comp Physiol. 2015;309(12):R1540-R1545.

138. Spronck B, Avolio AP, Tan I, Butlin M, Reesink KD, Delhaas T. Arterial stiffness index beta and cardio-ankle vascular index inherently depend on blood pressure but can be readily corrected. $J$ Hypertens. 2017;35(1):98-104.

139. Segers P. A lesson in vigilance: pressure dependency of a presumed pressure-independent index of arterial stiffness. J Hypertens. 2017;35(1):33-35.

140. Shirai K, Shimizu K, Takata M, Suzuki K. Independency of the cardioankle vascular index from blood pressure at the time of measurement. J Hypertens. 2017;35(7):1521-1523.

141. Grillo A, Salvi P. Cardio-ankle vascular index and carotidfemoral pulse wave velocity: limits and strengths. $J$ Hypertens. 2018;36(4):759-764

142. Obata Y, Mizogami M, Singh S, et al. Ejection time: influence of hemodynamics and site of measurement in the arterial tree. Hypertens Res. 2017;40(9):811-818.

143. Ueyama K, Miyata M, Kubozono T, et al. Noninvasive indices of arterial stiffness in hemodialysis patients. Hypertens Res. 2009;32(8):716-720.

144. Takizawa $\mathrm{C}$, Ito $\mathrm{M}$, Iwasa $\mathrm{M}$, et al. [Clinical significance of ABI by measuring bilateral arms in hemodialysis patients]. Japanese Association of Cardiovascular Intervention and Therapeutic (1884-0027)5, Suppl.I Page 736(2013.05). Japanese.

145. Verberk WJ, Kessels AG, Thien T. Blood pressure measurement method and inter-arm differences: a meta-analysis. Am J Hypertens. 2011;24(11):1201-1208.

146. Van Bergen FH, Weatherhead DS, Treloar AE, Dobkin AB, Buckley JJ. Comparison of indirect and direct methods of measuring arterial blood pressure. Circulation. 1954;10(4):481-490.

147. Gourdeau M, Martin R, Lamarche Y, Tétreault L. Oscillometry and direct blood pressure: a comparative clinical study during deliberate hypotension. Can Anaesth Soc J. 1986;33(3 Pt 1):300-307.

148. Ribezzo S, Spina E, di Bartolomeo S, Sanson G. Noninvasive techniques for blood pressure measurement are not a reliable alternative to direct measurement: a randomized crossover trial in ICU. Scientific World J. 2014;2014:353628

149. Tsushima M, Kyotani S, Nakano S, et al. [Diagnosis of early stages of atherosis and sclerosis of carotid artery and risk factors. The pulse wave velocity(PWV), ankle-brachial index(ABI), and the intima-media complex thickness(IMT) in inhabitants]. JJCDP. 2002;37:117-124. Japanese.

150. Takaki A, Ogawa H, Wakeyama T, et al. Cardio-ankle vascular index is superior to brachial-ankle pulse wave velocity as an index of arterial stiffness. Hypertens Res. 2008;31(7):1347-1355. 
151. Ichihara A, Yamashita N, Takemitsu T, et al. Cardio-ankle vascular index and ankle pulse wave velocity as a marker of arterial fibrosis in kidney failure treated by hemodialysis. Am J Kidney Dis. 2008;52(5):947-955.

152. Endes S, Caviezel S, Dratva J, et al. Reproducibility of oscillometrically measured arterial stiffness indices: Results of the SAPALDIA 3 cohort study. Scand J Clin Lab Invest. 2015;75(2):170-176.

153. Miyoshi T, Ito H, Horinaka S, Shirai K, Higaki J, Orimio H. Protocol for Evaluating the Cardio-Ankle Vascular Index to Predict Cardiovascular Events in Japan: A Prospective Multicenter Cohort Study. Pulse. 2017;4(Suppl 1):11-16.

154. García-Ortiz L, Recio-Rodríguez JI, Mora-Simón S, et al. Vascular structure and function and their relationship with health-related quality of life in the MARK study. BMC Cardiovasc Disord. 2016;16:95.

155. Gomez-Sanchez L, Garcia-Ortiz L, Patino-Alonso MC, et al. The Association Between the Cardio-ankle Vascular Index and Other Parameters of Vascular Structure and Function in Caucasian Adults: MARK Study. J Atheroscler Thromb. 2015;22(9):901-911.
156. Topouchian J, Labat C, Gautier S, et al. Effects of metabolic syndrome on arterial function in different age groups: the Advanced Approach to Arterial Stiffness study. J Hypertens. 2018;36(4):824-833.

157. Fukui T, Abe Y, Yasuda T, Yoshitaka S. [Comparison of the Measurements of Brachial-Ankle Pulse Wave Velocity (baPWV) and Cardioankle Vascular Index (CAVI) as an Examination for Aortic Stiffness]. Ningen Dock. 2008;23:70-76. Japanese.

158. Ato D, Sawayama T. Factors associated with high brachial-ankle pulse wave velocity in non-hypertensive and appropriately treated hypertensive patients with atherosclerotic risk factors. Vasc Health Risk Manag. 2017;13:383-392.

159. Kim T, Lee CS, Lee SD, et al. Impacts of comorbidities on the association between arterial stiffness and obstructive sleep apnea in the elderly. Respiration. 2015;89(4):304-311.

160. Zheng Y, Li Z, Shu H, Liu M, Chen Z, Huang J. Relationship between sum of the four limbs' pulse pressure and brachial-ankle pulse wave velocity and atherosclerosis risk factors in Chinese adults. Biomed Res Int. 2015;2015:434516-7. 


\section{Supplementary material}

Table SI Logistic regression analysis after redefining three studies (five studies in total)

\begin{tabular}{|c|c|c|c|c|}
\hline \multirow[b]{2}{*}{ Covariates } & \multicolumn{2}{|l|}{ Univariate } & \multicolumn{2}{|l|}{ Multivariate } \\
\hline & OR $(95 \% \mathrm{CI})$ & $P$-value & OR $(95 \% \mathrm{Cl})$ & $P$-value \\
\hline baPWV (yes = l) & $2.87(0.92-9.03)$ & 0.071 & $3.91(0.95-16.10)$ & 0.059 \\
\hline Exclusion of ASO/PAD (yes $=1$ ) & $4.68(1.73-12.70)$ & 0.003 & $4.01(1.22-13.20)$ & 0.022 \\
\hline Dialysis population (yes $=\mathrm{I}$ ) & $0.19(0.06-0.60)$ & 0.005 & $0.27(0.07-1.00)$ & 0.049 \\
\hline Age (years) & $0.99(0.93-1.07)$ & 0.87 & & \\
\hline Male gender $(\%)$ & $1.00(0.97-1.03)$ & 0.97 & & \\
\hline Follow-up period (years) & $1.00(0.78-1.27)$ & 0.97 & & \\
\hline Log (number of patients) & $14.00(3.13-62.80)$ & 0.0006 & $8.42(1.75-40.50)$ & 0.008 \\
\hline
\end{tabular}

Notes: baPWV $=\mathrm{I}$ or $\mathrm{CAVI}=0$ is used as a binary variate. The "success or failure" in the studies simultaneously comparing baPWV and CAVI was defined for each index. Thus, the total number of the included studies is 85.

Abbreviations: ASO, arteriosclerosis obliterans; baPWV, brachial-ankle pulse wave velocity; CAVI, cardio-ankle vascular index; PAD, peripheral arterial disease.

\section{Publish your work in this journal}

Vascular Health and Risk Management is an international, peerreviewed journal of therapeutics and risk management, focusing on concise rapid reporting of clinical studies on the processes involved in the maintenance of vascular health; the monitoring, prevention and treatment of vascular disease and its sequelae; and the involvement of metabolic disorders, particularly diabetes. This journal is indexed on PubMed Central and MedLine. The manuscript management system is completely online and includes a very quick and fair peer-review system, which is all easy to use. Visit http://www.dovepress.com/ testimonials.php to read real quotes from published authors. 